\title{
Emergent Hydrodynamics in Integrable Quantum Systems Out of Equilibrium
}

\author{
Olalla A. Castro-Alvaredo, ${ }^{1}$ Benjamin Doyon, ${ }^{2}$ and Takato Yoshimura ${ }^{2}$ \\ ${ }^{1}$ Department of Mathematics, City, University of London, \\ Northampton Square, London EClV OHB, United Kingdom \\ ${ }^{2}$ Department of Mathematics, King's College London, Strand, London WC2R 2LS, United Kingdom \\ (Received 12 July 2016; revised manuscript received 22 September 2016; published 27 December 2016)
}

Understanding the general principles underlying strongly interacting quantum states out of equilibrium is one of the most important tasks of current theoretical physics. With experiments accessing the intricate dynamics of many-body quantum systems, it is paramount to develop powerful methods that encode the emergent physics. Up to now, the strong dichotomy observed between integrable and nonintegrable evolutions made an overarching theory difficult to build, especially for transport phenomena where spacetime profiles are drastically different. We present a novel framework for studying transport in integrable systems: hydrodynamics with infinitely many conservation laws. This bridges the conceptual gap between integrable and nonintegrable quantum dynamics, and gives powerful tools for accurate studies of spacetime profiles. We apply it to the description of energy transport between heat baths, and provide a full description of the current-carrying nonequilibrium steady state and the transition regions in a family of models including the Lieb-Liniger model of interacting Bose gases, realized in experiments.

DOI: 10.1103/PhysRevX.6.041065

\section{INTRODUCTION}

Many-body quantum systems out of equilibrium give rise to some of the most important challenges of modern physics [1]. They have received a lot of attention recently, with experiments on quantum heat flows [2,3], generalized thermalization [4,5], and light-cone effects [6]. The leading principle underlying nonequilibrium dynamics is that of local transport carried by conserved currents. Deeper understanding can be gained from studying nonequilibrium, current-carrying steady states, especially those emerging from unitary dynamics [7]. This principle gives rise to two seemingly disconnected paradigms for manybody quantum dynamics. On the one hand, taking into account only few conservation laws, emergent hydrodynamics [8-12] offers a powerful description where the physics of fluids dominates [13-18]. On the other hand, in integrable systems, the infinite number of conservation laws is known to lead to generalized thermalization [19-21] (there are many fundamental works on the subject; see the review [22]), and the presence of quasilocal charges has been shown to influence transport [23,24] (see the review [25]). However, except at criticality $[26,27]$ (see the review [28]), no general many-body emergent dynamics has been proposed in the integrable case; with the available frameworks, these two paradigms seem difficult to bridge. The

Published by the American Physical Society under the terms of the Creative Commons Attribution 3.0 License. Further distribution of this work must maintain attribution to the author(s) and the published article's title, journal citation, and DOI.
Subject Areas: Nonlinear Dynamics,

Quantum Physics, Statistical Physics study of prethermalization or prerelaxation under small integrability breaking [22,28-30], the elusive quantum Kolmogorov-Arnold-Moser theorem [31,32], the development of perturbation theory for nonequilibrium states, and the exact treatment of nonequilibrium steady states and of nonhomogeneous quantum dynamics in unitary interacting integrable models remain difficult problems.

In this paper, using the recent advances on generalized thermalization and developing further aspects of integrability, we propose a solution to such problems by deriving a general theory of hydrodynamics with infinitely many conservation laws. The theory, applicable to a large integrability class, is derived solely from the fundamental tenet of emerging hydrodynamic: local entropy maximization (often referred to as local thermodynamic equilibrium) [33-37]. Focusing on quantum field theory (QFT) in one space dimension, we then study a family of models that include the paradigmatic Lieb-Liniger (LL) model [38] for interacting Bose gases, explicitly realized in experiments $[4,5,39-$ 41]. We concentrate on far-from-equilibrium states driven by heat baths in the partitioning protocol $[7,26,27,42]$ (see Fig. 1). We provide currents and full space-time profiles which are in principle experimentally accessible, beyond linear response and for arbitrary interaction strengths. We make contact with the physics of rarefaction waves, and with the concept of quasiparticle underlying integrable dynamics.

\section{SETUP}

Let two semi-infinite halves (which we refer to as the left and right reservoirs) of a homogeneous, short-range 


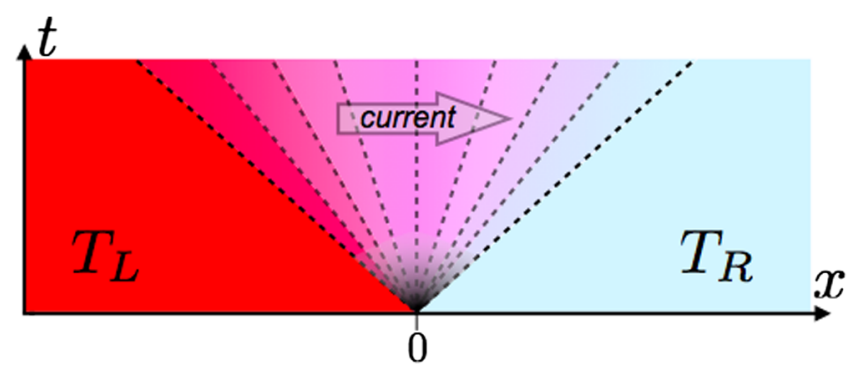

FIG. 1. The partitioning protocol. With ballistic transport, a current emerges after a transient period. Dotted lines represent different values of $\xi=x / t$. If a maximal velocity exists (e.g., due to the Lieb-Robinson bound), initial reservoirs are unaffected beyond it (light-cone effect). The steady state lies at $\xi=0$.

one-dimensional quantum system be independently thermalized, say, at temperatures $T_{L}$ and $T_{R}$. Let this initial state $\langle\cdots\rangle_{\text {ini }}$ be evolved unitarily with the Hamiltonian $H$ representing the full homogeneous system. One may then investigate the steady state that occurs at large times (see, e.g., Ref. [28]),

$\mathcal{O}^{\text {sta }}:=\lim _{t \rightarrow \infty}\left\langle e^{i H t} O e^{-i H t}\right\rangle_{\text {ini }}, \quad O$ local observable.

If the limit exists, it is a maximal-entropy steady state involving, in principle, all (quasi)local conserved charges of the dynamics $H$ [see Eq. (4)]. Generically, the dynamics only admits a single conserved quantity, $H$ itself: this means that, due to diffusive processes, ordinary Gibbs thermalization occurs. However, when conserved charges exist that are odd under time reversal, the steady state may admit nonzero stationary currents. This indicates the presence of ballistic transport, and the emergence of a current-carrying state that is far from equilibrium (breaking time-reversal symmetry). This is the partitioning protocol for building nonequilibrium steady states. See Fig. 1.

The study of such nonequilibrium steady states has received a large amount of attention recently (see Ref. [28] and references therein). They form a uniquely interesting set of states: they are simple enough to be theoretically described, yet encode nontrivial aspects of nonequilibrium physics. They naturally occur in the universal region near criticality described by QFT, where ballistic transport emerges thanks to continuous translation invariance, and in integrable systems, where it often arises thanks to the infinite family of conservation laws.

The works in Refs. [26,27] open the door to the study of nonequilibrium steady states at strong-coupling critical points with unit dynamical exponent, obtaining, in particular, the full universal time evolution. The steady state was found to be homogeneous within a light cone, with the energy current being

$$
\mathcal{L}^{\text {sta }}=\frac{\pi c k_{B}^{2}}{12 \hbar}\left(T_{L}^{2}-T_{R}^{2}\right)
$$

where $c$ is the central charge of the conformal field theory (CFT) (below, we set $k_{B}=\hbar=1$ ). This result arises from the independent thermalization of emerging left- and right-moving energy carriers (chiral separation). It was numerically verified [43] and agrees with recent heat-flow experiments [2]. It was generalized using hydrodynamic methods to higher-dimensional critical points $[13,14,17,18]$ and to deviations from criticality $[15,16,18]$. Under conditions that are fulfilled in universal near-critical regions, inequalities that generalize Eq. (2) can be derived [28,44] (here with unit Lieb-Robinson velocity [45]):

$$
\frac{e^{L}-e^{R}}{2} \geq j^{\text {sta }} \geq \frac{k^{L}-k^{R}}{2}
$$

where $e^{L, R}$ and $\ell^{L, R}$ are, respectively, the energy densities and the pressures in the left and right reservoirs [46].

Many further results exist in free-particle models (see Ref. [28] and references therein), where independent thermalization of right and left movers still holds. In contrast, however, only conjectures and approximations are available for interacting integrable models [47-49]. In addition, a striking dichotomy is observed between integrable situations and hydrodynamic-based results: for instance, conformal hydrodynamics is expected to emerge in strong-coupling CFT [13,14], leading to shock structures, but generically fails in free-particle conformal models [50], where transition regions are smooth. This points to the stark effect of integrability on nonequilibrium quantum dynamics, still insufficiently understood with available techniques.

\section{EMERGING HYDRODYNAMICS IN QUANTUM SYSTEMS}

Let us recall the basic concepts underlying the hydrodynamic description of many-body quantum systems, and its use in the setup described above (similar concepts exist in many-body classical systems).

Let $Q_{i}, i \in\{1,2, \ldots, N\}$, be local conserved quantities in involution. These are integrals of local densities $q_{i}(x, t)$, and the conservation laws take the form $\partial_{t} q_{i}(x, t)+$ $\partial_{x} j_{i}(x, t)=0$, where $j_{i}$ are the associated local currents. A Gibbs ensemble is a maximal-entropy ensemble under conditions fixing all averaged local conserved densities. It is described by a density matrix

$$
\rho=e^{-\sum_{i} \beta_{i} Q_{i}} / \operatorname{Tr}\left[e^{-\sum_{i} \beta_{i} Q_{i}}\right],
$$

where $\beta_{i}$ are the associated potentials. For instance, $Q_{1}$ is taken as the Hamiltonian, and $\beta_{1}$ is the inverse temperature. We denote $\beta=\left(\beta_{1}, \beta_{2}, \ldots, \beta_{N}\right)$ the vector representing this state, and $\langle\cdots\rangle_{\beta}$ the averages.

Clearly, the Gibbs averages of local densities $q_{i}=\left\langle q_{i}\right\rangle_{\beta}$ (these are independent of space and time by homogeneity 
and stationarity) may be seen as defining a map from states to averages, $\beta \mapsto q$. This is expected to be a bijection: the set of averages fully determines the set of potentials. Therefore, the current averages $\dot{z}_{i}=\left\langle j_{i}\right\rangle_{\underline{\beta}}$ are functions of the density averages:

$$
\underline{\dot{L}}=\underline{\mathcal{F}}(\underline{q}) .
$$

These are the equations of state, and are model dependent. The averages $\underline{q}$ can be generated by differentiation of the (specific, dimensionless) free energy $f_{\beta}$. Similarly, one can show [28] (see Appendix A) that there exists a function $g_{\underline{\beta}}$ that, likewise, generates the currents,

$$
\underline{q}=\nabla_{\underline{\beta}} f_{\underline{\beta}}, \quad \underline{\dot{j}}=\nabla_{\underline{\beta}} g_{\underline{\beta}} .
$$

A hydrodynamics description of quantum dynamics is expected to emerge at large space-time scales. This has been exploited, in the present setup, in Refs. [13-18]. The emergence of hydrodynamics is solely based on the assumption of local entropy maximization (or local thermodynamic equilibrium) [51]. Technically, this is the assumption that averages of local quantities $\langle O(x, t)\rangle$ tend uniformly enough, at large times, to averages evaluated in local Gibbs ensembles $\langle O\rangle_{\beta(x, t)}$ with space-time dependent potentials $\underline{\beta}(x, t)$. Physically, this is a consequence of separation of scales, as follows (see, for instance, Ref. [37]).

Assume that, after some time, physical properties vary only on space-time scales that are much larger than microscopic scales. This may be referred to as the "local relaxation time." From that time on, microscopic processes such as particle collisions or intersite interactions give rise to fast, local relaxation: the reaching of a (approximate) steady state on space-time scales small compared to variations but large enough for thermodynamics to be applicable. By Boltzmann's phase-space argument, these local steady states are obtained from entropy maximization, and as usual maximization is under the conditions provided by conservation laws (properties of the microscopic dynamics). That is, on each space-time "fluid cell" a Gibbs state is (very nearly) reached. Neighboring Gibbs states are different, but their variations are small. This is local entropy maximization.

Assume local entropy maximization. On each fluid cell, the Gibbs state is initially characterized by the values of the conserved densities at the local-relaxation time. The large-scale dynamics is thereon obtained from conservation laws, as follows. Consider microscopic conservation in integral form, $\int_{x_{1}}^{x_{2}} d x\left[q_{i}\left(x, t_{2}\right)-q_{i}\left(x, t_{1}\right)\right]+$ $\int_{t_{1}}^{t_{2}} d t\left[j_{i}\left(x_{2}, t\right)-j_{i}\left(x_{1}, t\right)\right]=0$. Since averages of densities and currents, after the local relaxation time, take the form $\left\langle q_{i}(x, t)\right\rangle=\left\langle q_{i}\right\rangle_{\underline{\beta}(x, t)}$ and $\left\langle j_{i}(x, t)\right\rangle=\left\langle j_{i}\right\rangle_{\underline{\beta}(x, t)}$ uniformly enough, we have

$$
\int_{x_{1}}^{x_{2}} d x\left[\underline{q}\left(x, t_{2}\right)-\underline{q}\left(x, t_{1}\right)\right]+\int_{t_{1}}^{t_{2}} d t\left[\underline{\dot{j}}\left(x_{2}, t\right)-\underline{\dot{j}}\left(x_{1}, t\right)\right]=0,
$$

where $\underline{q}(x, t)=\langle\underline{q}\rangle_{\underline{\beta}(x, t)}$ and $\underline{\dot{j}}(x, t)=\langle\underline{j}\rangle_{\hat{\beta}(x, t)}$. Here, integrals may be taken to cover a macroscopic number of fluid cells: these become macroscopic conservation equations. Macroscopic conservation equations can be rewritten in differential form, with differentials representing small variations amongst fluid cells:

$$
\partial_{t} \underline{q}(x, t)+\partial_{x \underline{\mathcal{z}}}(x, t)=0 .
$$

These are the pure hydrodynamic (Euler-type) equations, representing the slow, large-scale quantum dynamics of conserved densities and currents flowing amongst neighboring cells.

The problem of emergence of hydrodynamics in manybody systems is one of the most important unsolved problems of modern mathematical physics. Although there are few proofs of emergence of hydrodynamics, there is strong evidence for the validity of emerging Euler equations in many situations; see Refs. [33-37] and the recent paper Ref. [52] for a study of emerging Euler equations in classical anharmonic chains.

Combined with the equations of state Eq. (5), Euler equations (8) give

$$
\partial_{t} \underline{q}(x, t)+J(\underline{q}(x, t)) \partial_{x} \underline{q}(x, t)=0,
$$

where $J(\underline{q}):=\nabla_{q} \dot{\mathcal{L}}$ is an $N$ by $N$ matrix, the Jacobian matrix of the transformation from densities to currents:

$$
J(\underline{q})_{i j}=\partial \mathcal{F}_{i}(\underline{q}) / \partial q_{j} .
$$

Equation (9) is the emergent pure hydrodynamic equation in quasilinear (or characteristic) form [12]. The complete model dependence, including all quantum effects, is encoded, besides the number $N$ of conserved quantities, in the Jacobian $J(\underline{q})$.

The density averages $\underline{q}$, like the potentials $\beta$, correspond to a set of state coordinates. One may choose any other set of state coordinates $\underline{n}$, with $\underline{q}=\underline{\mathcal{F}}^{q}(\underline{n})$ and $\underline{\dot{j}}=\underline{\mathcal{F}}^{\dot{ }}(\underline{n})$. A similar equation is obtained,

$$
\partial_{t} \underline{n}(x, t)+J(\underline{n}(x, t)) \partial_{x \underline{n}}(x, t)=0,
$$

where $J(\underline{n})=\left(\nabla_{\underline{n}} \underline{q}\right)^{-1} \nabla_{\underline{n}} \underline{\dot{j}}$. Observe that $J(\underline{n})$ and $J(\underline{q})$ are related to each other by a similarity transformation: $J(\underline{n})=\left.\left(\nabla_{\underline{n} \underline{\dot{z}}}\right)^{-1} J(\underline{q})\right|_{\underline{q}=\underline{\mathcal{F}}^{q}(\underline{n})} \nabla_{\underline{n} \underline{\dot{z}}}$. Therefore, the spectrum of $J(\underline{n})$ is independent of the choice of coordinates, 
and is a fundamental property of the model. We denote this spectrum by $\left\{v_{i}^{\text {eff }}(\underline{n}), i=1, \ldots, N\right\}$.

Choosing coordinates $\underline{n}$ that diagonalize $J(\underline{n})$, one obtains

$$
\partial_{t} n_{i}(x, t)+v_{i}^{\mathrm{eff}}(\underline{n}(x, t)) \partial_{x} n_{i}(x, t)=0 .
$$

These express the vanishing of the convective derivatives, representing the constancy of each fluid mode $n_{i}(x, t)$ on fluid cells. The eigenvalues $v_{i}^{\text {eff }}(\underline{n}(x, t))$ are, therefore, interpreted as the propagation velocities of these normal modes. The normal modes interact with each other only through the propagation velocities, which is generically a function of all state coordinates.

Let us now apply the above to the solution of the partitioning problem. For clarity of the following discussion, we come back to the $q$ coordinates (but it is easy to generalize to any coordinates $n$ ). Consider the large-scale limit $(x, t) \mapsto(a x, a t), a \rightarrow \infty$. Because Eq. (12) is invariant under this scaling, in the limit, if it exists, the solution is also invariant. Thus, we may assume self-similar solutions $\beta(x, t)=\beta(\xi)$, where $\xi=x / t$, and Eq. (12) becomes an eigenvalue equation:

$$
[J(\underline{q})-\xi \mathbf{1}] \partial_{\xi \underline{q}}=0 .
$$

The initial condition is determined by the state at the local relaxation time (at which the fluid-dynamics description starts to be valid). This state is unknown, as it depends on the full quantum dynamics, but its asymptotic at large $|x|$ is identical to that of the original state. In the large-scale solution, the initial condition $t \rightarrow 0^{+}$is implemented as asymptotic conditions as $\xi \rightarrow \pm \infty$. Therefore, it depends only on the asymptotic form of the initial state, and we impose

$$
\lim _{\xi \rightarrow \pm \infty} \underline{q}(\xi)=\lim _{x \rightarrow \pm \infty}\langle\underline{q}(x, 0)\rangle_{\text {ini }}
$$

In the present setup, these involve Gibbs states at potentials $\underline{\beta}^{R, L}$ :

$$
\lim _{x \rightarrow \pm \infty}\langle\underline{q}(x, 0)\rangle_{\text {ini }}=\langle\underline{q}\rangle_{\underline{\beta}^{R, L}}
$$

and the steady-state averages are given by

$$
\underline{q}^{\text {sta }}:=\underline{q}(\xi=0), \quad \underline{\dot{L}}^{\text {sta }}:=\underline{\dot{j}}(\xi=0) .
$$

The solution to the eigenvalue equation (13) and initial conditions Eq. (14) provides the exact large-scale asymptotic form of the full quantum solution, along any ray $x=$ $\xi t$ (see Fig. 1). The eigenvalue equation (13) represents the small changes of averages along various rays, due to the exchange of conserved charges amongst fluid cells. The set of eigenvalues of $J(\underline{q})$-the available propagation velocities in the state characterized by the averages $q$ form a finite, discrete set for finite $N$.

Solutions to Eqs. (13) and (14) are typically composed of regions of constant $q$ values separated by transition regions [12]. Transition regions may be of two types: either shocks (weak solutions), where $q$ values display finite jumps, or rarefaction waves, where they form a smooth solution to Eq. (13). Rarefaction waves, the most natural type of solution, cannot, generically, cover the full space between two reservoirs. Indeed, Eq. (13) specifies that the curve traced by the solution in the $q$ plane must at all points be tangent to an eigenvector of $J(\underline{q})$. Since eigenvectors-and available propagation velocities-form a discrete set, smooth variations of $q$ along the curve imply a unique choice of eigenvector at each point (except possibly at points where eigenvalues cross). Thus, the curve is completely determined by its initial point, and cannot join two arbitrary reservoir values. That is, in ordinary pure hydrodynamics, shocks are often required.

\section{HYDRODYNAMICS WITH INFINITELY MANY CURRENTS}

In integrable systems, there are infinitely many local conservation laws. In fact, this space is enlarged to that of "pseudolocal" conservation laws, where the densities $q_{i}(x, t)$ and currents $j_{i}(x, t)$ are supported on extended spacial regions with weight decaying fast enough away from $x$. This enlargement plays an important role in nonequilibrium quantum dynamics [20,21,23-25]. Under maximal-entropy principles, Gibbs states are then replaced by generalized Gibbs ensembles (GGE) [19,21,22]: formally, the limit $N \rightarrow \infty$ of the density matrix Eq. (4), involving all basis elements in the space of conserved pseudolocal charges. We choose $Q_{1}=H$ (the Hamiltonian) and $Q_{2}=P$ (the momentum operator).

Under the influence of infinitely many conservation laws, the picture of local entropy maximization is still expected to hold: all physical principles underlying it stay unchanged, the only difference being the use of GGEs instead of Gibbs ensembles. This, along with the emergence of self-similar solutions in the partitioning protocol, are our working hypotheses; see Appendix B for a discussion. The emergence of a generalized type of hydrodynamics was proven in the classical hard-rod problem [37,53], whose relation with the present quantum problem we will study in a future work. The emergence of self-similar solutions was observed numerically in the quantum $X X Z$ chain in Ref. [54]. In free-particle quantum models, hydrodynamic ideas and related semiclassical approximations, as well as ray-dependent local entropy maximization, were studied in various works; see Refs. [55-60].

Looking for a full solution to the infinity of Eqs. (9), (13), and (14), an appropriate choice of state variables is 
crucial. A powerful way is to recast them into the quasiparticle language underlying the thermodynamic Bethe ansatz (TBA) method for integrable systems [61]. Using this language, we derive the exact GGE equations of state and the ensuing generalized hydrodynamics equation. We determine the exact normal modes and propagation velocities, and obtain full ray-dependent solutions.

\section{A. GGE equations of state}

We assume that the spectrum of stable quasiparticles is composed of a single quasiparticle species of mass $m$ (see Appendix $\mathrm{C}$ for a many-particle generalization). The dispersion relation is encoded via a parametrization $E(\theta)$, $p(\theta)$ of the energy and momentum: in the relativistic case, $\theta$ is the rapidity, $E(\theta):=m \cosh (\theta), p(\theta):=m \sinh (\theta)$, and in the Galilean case, $\theta$ is the velocity, $E(\theta):=m \theta^{2} / 2$, $p(\theta):=m \theta$. In integrable models, scattering is elastic and factorizes into two-particle processes, and a model is fully specified by giving the elastic two-particle scattering amplitude $S\left(\theta_{1}-\theta_{2}\right)$. The differential scattering phase is defined as $\varphi(\theta)=-i d \log S(\theta) / d \theta$. We denote by $h_{i}(\theta)$ the oneparticle eigenvalue of the conserved charge $Q_{i}$; in particular, $h_{1}(\theta)=E(\theta)$ and $h_{2}(\theta)=p(\theta)$.

Let us first recall the basic ingredients of TBA. Three related quantities play important roles: the quasiparticle density $\rho_{p}(\theta)$, the state density $\rho_{s}(\theta)$, and the quasiparticle occupation number $n(\theta):=\rho_{p}(\theta) / \rho_{s}(\theta)$. The functions $\rho_{p}(\theta)$ and $n(\theta)$ are two different sets of state coordinates; each can be used to fully characterize the GGE. The former specifies all average densities in a simple way:

$$
q_{i}=\int d \theta \rho_{p}(\theta) h_{i}(\theta)
$$

This can, in fact, be seen as a definition of $\rho_{p}(\theta)$. Here and below, integrations are over $\mathbb{R}$.

As a consequence of interactions, quasiparticle and state densities are related to each other. Using the Bethe ansatz, one finds the following constitutive relation [61]:

$$
2 \pi \rho_{s}(\theta)=p^{\prime}(\theta)+\int d \alpha \varphi(\theta-\alpha) \rho_{p}(\alpha)
$$

where $p^{\prime}(\theta)=d p(\theta) / d \theta$. This relation gives rise to a nonlinear relation between the state coordinates $\rho_{p}(\theta)$ and $n(\theta)$. The transformation from the former to the latter is direct from the above definitions. In the opposite direction, the transformation is effected by

$$
2 \pi \rho_{p}(\theta)=n(\theta)\left(p^{\prime}\right)^{\mathrm{dr}}(\theta)
$$

where the "dressing" operation $h \mapsto h^{\mathrm{dr}}$ is defined by the solution to the linear integral equation:

$$
h^{\mathrm{dr}}(\theta)=h(\theta)+\int \frac{d \gamma}{2 \pi} \varphi(\theta-\gamma) n(\gamma) h^{\mathrm{dr}}(\gamma)
$$

The potentials $\beta$ can be recovered: the occupation number is related to the one-particle eigenvalue $w(\theta)=$ $\sum_{i} \beta_{i} h_{i}(\theta)$ of the charge $\sum_{i} \beta_{i} Q_{i}$ in the GGE Eq. (4) via the so-called pseudoenergy $\epsilon_{w}(\theta)[61,62]$ :

$$
\begin{aligned}
n(\theta) & =\frac{1}{1+\exp \left[\epsilon_{w}(\theta)\right]}, \\
\epsilon_{w}(\theta) & =w(\theta)-\int \frac{d \gamma}{2 \pi} \varphi(\theta-\gamma) \log \left(1+e^{-\epsilon_{w}(\gamma)}\right) .
\end{aligned}
$$

The above ingredients give exact average densities as functions of GGE states. However, they do not provide expressions for average currents as functions of state coordinates, and for equations of states. Hence, they are not sufficient in order to develop generalized hydrodynamics.

We solve this problem by obtaining the following expressions:

$$
q_{i}=\int \frac{d p(\theta)}{2 \pi} n(\theta) h_{i}^{\mathrm{dr}}(\theta), \quad \dot{z}_{i}=\int \frac{d E(\theta)}{2 \pi} n(\theta) h_{i}^{\mathrm{dr}}(\theta),
$$

where $h_{i}^{\mathrm{dr}}(\theta)$ is the dressed one-particle eigenvalue. These expressions emphasize the role of relativistic or Galilean symmetry: the sole difference between GGE averages of charge densities and currents is the integration measure, determined by the dispersion relation.

The first equation in Eq. (22) is well known and is a consequence of Eqs. (17) and (19). In integral-operator notation [with measure $d \theta /(2 \pi)$ ], the dressing operation is

$$
h^{\mathrm{dr}}=(1-\varphi \mathcal{N})^{-1} h,
$$

where $\mathcal{N}$ is diagonal with kernel $2 \pi n(\theta) \delta(\theta-\alpha)$, and $\varphi$ has kernel $\varphi(\theta-\alpha)$. Therefore, introducing the symmetric operator $\mathcal{U}=\mathcal{N}(1-\varphi \mathcal{N})^{-1}$ and the bilinear form $a \cdot b=\int d \theta /(2 \pi) a(\theta) b(\theta)$, we have

$$
q_{i}=h_{i} \cdot \mathcal{U} p^{\prime}=p^{\prime} \cdot \mathcal{U} h_{i}
$$

which leads to the first equation of Eq. (22).

The second equation in Eq. (22) is new. It may be proven, in the relativistic case, using relativistic crossing symmetry, and then obtained by the nonrelativistic limit in the Galilean case. In the relativistic case, crossing symmetry says that local currents $j_{i}$, in the cross channel, are local densities $q_{i}$; therefore, the current expression in Eq. (22) is obtained from that of the density under an appropriate exchange of energy and momentum. Let $\mathcal{C}$ be the crossing transformation $(x, t) \mapsto(i t,-i x)$, implemented on rapidities by $\theta \mapsto i \pi / 2-\theta$. Note that it squares to the identity $\mathcal{C}^{2}=1$. Let us denote by $q[h]$ and $j[h]$ the density and 
current operators, respectively, associated to a one-particle eigenvalue $h(\theta)$. Then, the statement that local currents $j_{i}$, in the cross channel, are local densities $q_{i}$ translates into $\mathcal{C}(j[h])=i q\left[h^{C}\right]$, where $h^{C}(\theta)=h(i \pi / 2-\theta)$. Let us also denote by $\langle\mathcal{O}\rangle_{w}$ the average of observables $\mathcal{O}$ in the state characterized by $w(\theta)$. Then, $\langle\mathcal{C}(\mathcal{O})\rangle_{w}=\langle\mathcal{O}\rangle_{w^{c}}$, where $w^{C}(\theta)=w(i \pi / 2-\theta)$. Using $\langle j[h]\rangle_{w}=\langle\mathcal{C}(\mathcal{C}(j[h]))\rangle_{w}=$ $i\left\langle q\left[h^{c}\right]\right\rangle_{w^{c}}$ and the expression for $q_{i}=q\left[h_{i}\right]$ in Eq. (22), we obtain the expression for $\dot{j}_{i}=\dot{j}_{i}\left[h_{i}\right]$. An alternative proof, using the machinery of integrable systems, is presented in Appendix D.

Expressions (22) have interesting consequences. First, using $\dot{z}_{i}=h_{i} \cdot \mathcal{U} E^{\prime}$, where $E^{\prime}(\theta)=d E(\theta) / d \theta$, in Eq. (22), the average current may also be written in terms of a current spectral density $\rho_{c}(\theta)$ :

$$
\dot{\mathcal{z}}_{i}=\int d \theta \rho_{c}(\theta) h_{i}(\theta)
$$

which takes the forms

$$
2 \pi \rho_{c}(\theta)=n(\theta)\left(E^{\prime}\right)^{\mathrm{dr}}(\theta)=2 \pi v^{\mathrm{eff}}(\theta) \rho_{p}(\theta) .
$$

Here, $v^{\text {eff }}(\theta)$ is the effective velocity, defined by

$$
v^{\mathrm{eff}}(\theta):=\frac{\left(E^{\prime}\right)^{\mathrm{dr}}(\theta)}{\left(p^{\prime}\right)^{\mathrm{dr}}(\theta)}
$$

The effective velocity depends on the state via the occupation number entering the dressing operation, and brings out the quasiparticle interpretation of the current expression: since $\rho_{c}(\theta)=v^{\text {eff }}(\theta) \rho_{p}(\theta)$, quasiparticles are seen as moving at effective velocities $v^{\text {eff }}(\theta)$, influenced by the state in which they move.

Second, one may extract explicit GGE equations of state from expressions (22). The equations of states are necessary and sufficient relations between densities and currents, guaranteeing the existence of $n(\theta)$ such that both relations in Eq. (22) hold for all $h_{i}(\theta)$. Assume that $q_{i}$ and $\dot{z}_{i}$ are averages in a state, not necessarily a GGE. In complete generality, both are linear functionals of $h(\theta)$; hence, we may still write Eqs. (17) and (25) for some quasiparticle density $\rho_{p}(\theta)$ and current spectral density $\rho_{c}(\theta)$. GGE equations of states can, therefore, be written as relations between $\rho_{p}(\theta)$ and $\rho_{c}(\theta)$, necessary and sufficient for the existence of $n(\theta)$ such that Eqs. (22) hold. One can show that these relations are

$$
\frac{\rho_{c}(\theta)}{\rho_{p}(\theta)}=\frac{E^{\prime}(\theta)+\int d \alpha \varphi(\theta-\alpha) \rho_{c}(\alpha)}{p^{\prime}(\theta)+\int d \alpha \varphi(\theta-\alpha) \rho_{p}(\alpha)} .
$$

These relations are independent of the state: they hold in any GGE, in the model described by the differential scattering phase $\varphi(\theta-\alpha)$. They characterize the set of doublets of functions $\left(\rho_{p}, \rho_{c}\right)$ describing available GGEs for this integrable model. The proof of Eq. (28) is obtained by isolating $n(\theta)$ in both Eqs. (19) and (26), in the forms $2 \pi\left(\mathcal{N}^{-1}-\varphi\right) \rho_{p}=p^{\prime}$ and $2 \pi\left(\mathcal{N}^{-1}-\varphi\right) \rho_{c}=E^{\prime}$, and equating the resulting expressions.

Finally, recalling Eq. (26), the left-hand side of Eq. (28) is $v^{\text {eff }}(\theta)$. Simple manipulations of Eq. (28) then give a linear integral equation for the effective velocity $v^{\text {eff }}(\theta)$ in terms of quasiparticle densities:

$v^{\mathrm{eff}}(\theta)=v^{\mathrm{gr}}(\theta)+\int d \alpha \frac{\varphi(\theta-\alpha) \rho_{p}(\alpha)}{p^{\prime}(\theta)}\left[v^{\mathrm{eff}}(\alpha)-v^{\mathrm{eff}}(\theta)\right]$,

where $v^{\mathrm{gr}}(\theta)=E^{\prime}(\theta) / p^{\prime}(\theta)$ is the group velocity. In this form, the equations of state of integrable systems are seen as equations specifying an effective velocity of quasiparticles, as a modification of the group velocity.

We note that the effective velocity we derive here agrees with that proposed in Ref. [63]. [Note that in Ref. [63] $v^{\text {eff }}(\theta)$ is written in a form similar to, but different from, Eq. (27), using a different definition of dressing.] This is interesting, as our derivation is based on comparing current spectral density to quasiparticle density, while the concept proposed in Ref. [63] is based on stationary-phase arguments.

\section{B. Generalized hydrodynamics}

The basic equation of generalized pure hydrodynamics is derived from Eq. (8) along with the quasiparticle expressions Eqs. (17) and (25). The fact that the space of pseudolocal charges is complete [21] suggests that these hold for a complete set of functions $h_{i}(\theta)$, and, thus (here and below we suppress explicit $x, t$ dependences for lightness of notation),

$$
\partial_{t} \rho_{p}(\theta)+\partial_{x} \rho_{c}(\theta)=0 .
$$

Using the equations of state Eq. (28), this is an integrodifferential system for the space-time-dependent state characterized by the particle densities $\rho_{p}(\theta)$.

Alternatively, using the effective-velocity formulation Eqs. (26) and (29), Eq. (30) may be written as

$$
\partial_{t} \rho_{p}(\theta)+\partial_{x}\left[v^{\mathrm{eff}}(\theta) \rho_{p}(\theta)\right]=0 .
$$

This is the conservation form of generalized hydrodynamics. It is a density-type conservation equation, and it identifies $\rho_{p}(\theta)$ as a conserved fluid density.

The state coordinates $\rho_{p}(\theta)$ are, however, not the most convenient. We show that the occupation numbers $n(\theta)$ diagonalize the Jacobian $J(\underline{n})$ in the quasilinear form Eq. (11): the space-time-dependent occupation number $n(\theta)$ satisfies the following integro-differential system, the vanishing of the convective derivative of $n(\theta)$ : 


$$
\partial_{t} n(\theta)+v^{\mathrm{eff}}(\theta) \partial_{x} n(\theta)=0 .
$$

Here, Eq. (27) may be used to express the effective velocity in terms of $n(\theta)$. Hence, $n(\theta)$ are the normal modes of generalized hydrodynamics, and further, the eigenvaluesthe propagation velocities-are exactly the effective velocities $v^{\text {eff }}(\theta)$.

The proof of Eq. (32) is as follows. Using the integraloperator relations $2 \pi \rho_{p}=\mathcal{U} p^{\prime}$ and $2 \pi \rho_{c}=\mathcal{U} E^{\prime}$, we have $\left(\partial_{t} \mathcal{U}\right) p^{\prime}+\left(\partial_{x} \mathcal{U}\right) E^{\prime}=0$. Taking derivatives, $\partial_{x, t} \mathcal{U}=$ $(1-\mathcal{N} \varphi)^{-1}\left(\partial_{x, t} \mathcal{N}\right)(1-\varphi \mathcal{N})^{-1}$, and we obtain

$$
\partial_{t} \mathcal{N}(1-\varphi \mathcal{N})^{-1} p^{\prime}+\partial_{x} \mathcal{N}(1-\varphi \mathcal{N})^{-1} E^{\prime}=0,
$$

which gives Eq. (32) using Eq. (23).

Observe that, using Eqs. (31) and (32), it is simple to show that the state density $\rho_{s}(\theta)$ as well as the hole density $\rho_{h}(\theta):=\rho_{s}(\theta)-\rho_{p}(\theta)$ also satisfy the same density-type conservation equation (31) (this was noted in Ref. [64]). Further, as a consequence, the entropy density [61],

$s(\theta):=\rho_{s}(\theta) \log \rho_{s}(\theta)-\rho_{p}(\theta) \log \rho_{p}(\theta)-\rho_{h}(\theta) \log \rho_{h}(\theta)$,

also satisfies this conservation equation, $\partial_{t} s(\theta)+$ $\partial_{x}\left[v^{\text {eff }}(\theta) s(\theta)\right]=0$. Conservation of entropy density is a fundamental property of perfect fluids, as no viscosity effects are taken into account.

In the large-scale limit, the equation for the raydependent ( $\xi$-dependent) occupation number $n(\theta)$ simplifies to

$$
\left[v^{\mathrm{eff}}(\theta)-\xi\right] \partial_{\xi} n(\theta)=0
$$

This is the eigenvalue equation (13) in the occupationnumber coordinates (which diagonalize the Jacobian), and its solution gives $q(\xi)$ and $\dot{j}(\xi)$ via Eqs. (22) and (20).

One can show that the solution for the nonequilibrium, ray-dependent occupation number $n(\theta)$ is the discontinuous function

$$
n(\theta)=n^{L}(\theta) \Theta\left(\theta-\theta_{\star}\right)+n^{R}(\theta) \Theta\left(\theta_{\star}-\theta\right),
$$

where $\Theta(\cdots)$ is Heavyside's step function. The position of the discontinuity $\theta_{\star}$ depends on $\xi$ and is self-consistently determined by $v^{\text {eff }}\left(\theta_{\star}\right)=\xi$; equivalently, it is the zero of the dressed, boosted momentum $p_{\xi}(\theta):=p(\theta-\eta)$, where $\xi=\tanh \eta$ (relativistic case) or $\xi=\eta$ (Galilean case),

$$
p_{\xi}^{\mathrm{dr}}\left(\theta_{\star}\right)=0 .
$$

The GGE occupation numbers $n^{L, R}(\theta)$ entering Eq. (35) guarantee that the asymptotic conditions on $\xi$ correctly represent the asymptotic baths as per Eq. (14). They are obtained using Eq. (21) with $w=w^{L, R}(\theta)$ the one-particle eigenvalues characterizing the GGE of the left and right asymptotic reservoirs; for instance, with reservoirs at temperatures $T_{L, R}$, we have $w^{L, R}(\theta)=T_{L, R}^{-1} E(\theta)$.

Indeed, the solution Eq. (35) of the scaled problem holds since $v^{\text {eff }}(\theta)$ is monotonic and covers the full range of $\theta$ (which is $[-1,1]$ in the relativistic case and $\mathbb{R}$ in the Galilean case); therefore, there is a unique solution to $v^{\text {eff }}(\theta)=\xi$, and, thus, a unique jump, and $\theta_{\star}$ is monotonic with $\xi$; hence, the asymptotic conditions are correctly implemented.

The system of integral equations (22), (20), (35), and (36) can be solved numerically using Mathematica, yielding extremely accurate results. Integral equations in Eqs. (21) and (20) can be solved iteratively, a procedure that converges fast [61]. The hydrodynamic solution is obtained by first constructing the thermal occupation numbers $n^{L, R}(\theta)$ [Eq. (21)]. Then, the nonequilibrium occupation number is evaluated by solving the system equations (35) and (36): one first chooses $\theta_{\star}=\eta$ in order to construct $n(\theta)$, and then evaluates $p_{\xi}^{\mathrm{dr}}(\theta)$. The zero of $p_{\xi}^{\mathrm{dr}}(\theta)$ is numerically found-we observe that $p_{\xi}^{\mathrm{dr}}(\theta)$ always has a single zero. The process is repeated until the zero is stable-we observe that this is a convergent procedure. Finally, the nonequilibrium occupation number is used in Eqs. (22) and (20). The solving time increases slowly with the numerical precision demanded; thus, this allows arbitrary-precision results.

The solution we present may be interpreted as a single space-covering rarefaction wave, in the sense that it is a solution to the eigenvalue equation (13) where all physical observables $q_{i}, \dot{z}_{i}$ are continuous and interpolate between the two reservoirs. With relativistic dispersion relation, the solution is smooth within the light cone, beyond which the states are constant and equal to the initial baths' states; while in the Galilean case, the solution is generically smooth on the whole space. In this solution, every normal mode $n(\theta)$, seen as a function of $\xi$ for fixed $\theta$, is discontinuous exactly at its propagation velocity. Every normal mode therefore displays a "contact discontinuity" (a discontinuity without entropy production) [12]. Hence, the rarefaction wave may be seen as being composed of infinitely many contact discontinuities. In contrast to the finite-dimensional case, this single rarefaction wave can account for generic reservoirs, and no shocks need to develop. This is because in the infinite-dimensional case, the eigenvalues of $J(\underline{n})$ form a continuum: all propagation velocities $v^{\text {eff }}(\theta)$ are available as conserved charges guarantee a large number of stable excitations, providing an additional continuous parameter tuning the smooth state trajectory and guaranteeing its correct asymptotic-reservoir values. Since weak solutions (shocks) are not necessary to connect the asymptotic reservoirs, they do not appear. 


\section{ANALYSIS AND DISCUSSION}

Concentrating on pure thermal transport, we analyze the above general system of equations for two related models: the relativistic integrable sinh-Gordon model and its nonrelativistic limit [65,66], the (repulsive) Lieb-Liniger model. We also verify that our hydrodynamic equations reproduce the known results for the case of free particles.

\section{A. Relativistic sinh-Gordon model}

One of the simplest integrable relativistic QFTs with nontrivial interactions is the sinh-Gordon model. It is defined by the Lagrangian $[67,68]$

$$
\mathcal{L}_{\text {shG }}=\frac{1}{2}\left(\partial_{\mu} \phi\right)^{2}-\frac{m^{2}}{\beta^{2}} \cosh (\beta \phi),
$$

where $\phi$ is the sinh-Gordon field and $m$ is the mass of the single particle in the spectrum. The model is integrable and, therefore, the only nontrivial scattering matrix is that associated to two-particle scattering. It is given by [69-71]

$$
S(\theta)=\frac{\tanh \frac{1}{2}\left(\theta-\frac{i \pi B}{2}\right)}{\tanh \frac{1}{2}\left(\theta+\frac{i \pi B}{2}\right)} .
$$

The parameter $B \in[0,2]$ is the effective coupling constant, which is related to the coupling constant $\beta$ in the Lagrangian by

$$
B(\beta)=\frac{2 \beta^{2}}{8 \pi+\beta^{2}},
$$

under CFT normalization [72]. The $S$ matrix is obviously invariant under the transformation $B \rightarrow 2-B$, a symmetry that is also referred to as weak-strong coupling duality, as it corresponds to $B(\beta) \rightarrow B\left(\beta^{-1}\right)$ in Eq. (39). The point $B=1$ is known as the self-dual point. At the self-dual point the TBA differential scattering phase is simply

$$
\varphi_{\mathrm{shG}}(\theta)=-i \frac{d}{d \theta} \log S(\theta)=\frac{2}{\cosh \theta} .
$$

Contrary to the Lieb-Liniger model, which we discuss later, the general features of any quantities of interest in the sinh-Gordon model are very similar for any values of the coupling $B$. For this reason, in this paper we concentrate our analysis solely on the self-dual point in the understanding that similar results hold for other values of $B$.

We evaluate the energy density $e:=q_{1}$, energy current $\dot{j}:=\dot{j}_{1}$, and pressure $k:=\dot{j}_{2}$. Typical profiles are shown in Figs. 2 and 3. Figure 2 shows smooth interpolation within the light cone between the asymptotic baths at $\xi=-1$ and $\xi=1$ (the speed of light is set to 1). Figure 3 shows how, as temperatures rise, the current approaches the plateau [Eq. (2)] predicted by CFT [26,27]. Further, in

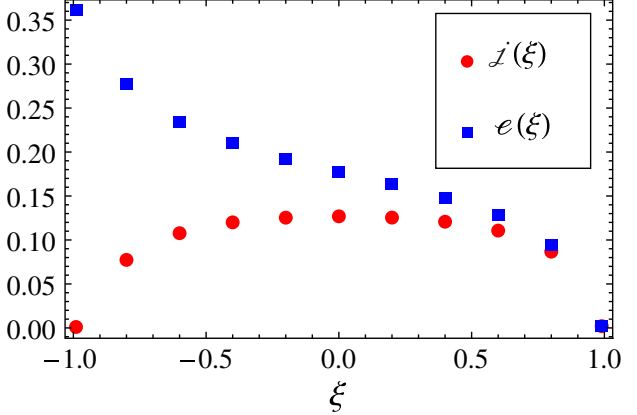

FIG. 2. The functions $j(\xi)$ (dots) and $e(\xi)$ (squares) for $\beta_{L}=1$ and $\beta_{R}=30$ in the sinh-Gordon model.

Fig. 4, the relative deviation of the steady-state current from its bounds [Eq. (3)] is shown. The bounds are extremely tight, pointing to the strength of this constraint and confirming that the proposed solution is correct. The bounds are indeed so tight that it is difficult to distinguish some points in parts of Fig. 4. To better appreciate this, we present the numerical values of the points displayed in Fig. 4 (divided by $\beta_{L}^{2}$ ) in Table I.

The numerical data are obtained by solving the integral equations recursively until convergence is reached. Sources of error are the discretization and finite range of $\theta$ for numerical integration. Adjusting the number of divisions and the range, we estimate the error to be less than $0.1 \%$.

\section{B. Lieb-Liniger model}

The Lieb-Liniger model, in the repulsive regime $(\lambda>0)$, can be regarded as a nonrelativistic limit of the sinh-Gordon model, as shown in Refs. $[65,66]$. The Hamiltonian of the model is given by

$$
H_{\mathrm{LL}}=\int d x\left(\frac{1}{2 m} \partial_{x} \psi^{\dagger} \partial_{x} \psi+\lambda \psi \psi^{\dagger} \psi^{\dagger} \psi \psi\right)
$$

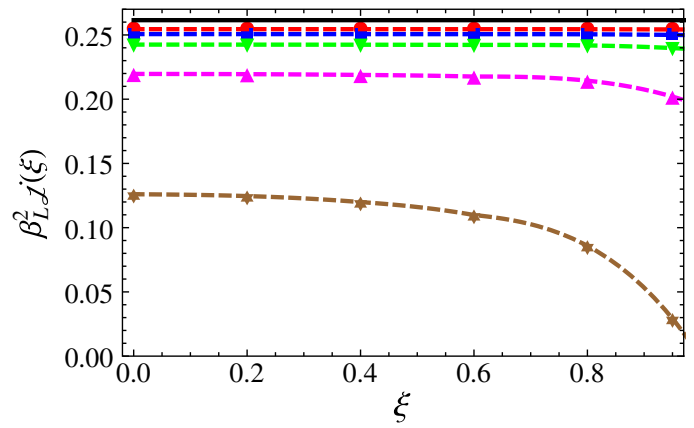

FIG. 3. The functions $\beta_{L}^{2} j(\xi>0)$ for $\beta_{R}=30 \beta_{L}$ and $\beta_{L}=10^{-p}$, with $p=0$ (stars), 1 (triangles), 2 (inverted triangles), 3 (squares), and 4 (circles). The continuous bold line represents the conformal value $\beta_{L}^{2} j(\xi)=\frac{\pi}{12}\left(1-\frac{1}{900}\right)$ which, as expected, is reached at high temperatures. Dashed curves are interpolations. 


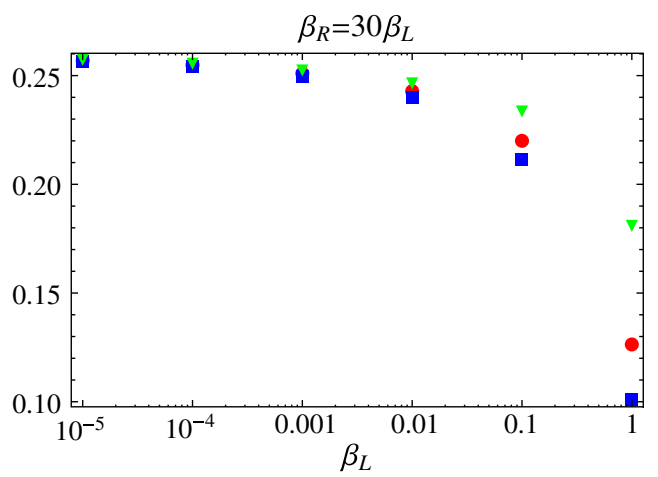

FIG. 4. Verification of the inequalities Eq. (3) in the sinhGordon model. Displayed are the functions $\beta_{L}^{2} \mathcal{L}^{\text {sta }}$ (circles), $\beta_{L}^{2}\left(e^{L}-e^{R}\right) / 2$ (triangles), and $\beta_{L}^{2}\left(\hbar^{L}-\hbar^{R}\right) / 2$ (squares).

This is obtained from the Hamiltonian of the sinh-Gordon model by a double limit,

$$
c \rightarrow \infty, \quad \beta \rightarrow 0 ; \quad \beta c=4 \sqrt{\lambda},
$$

where $c$ is the speed of light [which is implicit in Eq. (37)]. (This is the only equation in the present paper where the speed of light $c$ appears explicitly. Everywhere else $c$ denotes the central charge. As both are standard notations, we choose to use the notation $c$ for both.) This limit can be performed within the TBA formalism [66], and accordingly, the density and current averages $q_{i}, \dot{z}_{i}$ are given by Eq. (22), with the nonrelativistic dispersion relation. There, the occupation number is given by $n_{\mathrm{LL}}(\theta)=1 /\left(1+e^{\epsilon_{w}(\theta)}\right)$, and the pseudoenergy $\epsilon_{w}(\theta)$ and the dressed one-particle eigenvalues $h_{i}^{\mathrm{dr}}(\theta)$ are defined in the same manner as in Eqs. (21) and (20) (where $\theta=p / m$ is the velocity), with the scattering matrix given by

$$
S_{\mathrm{LL}}(\theta)=\frac{\theta-2 \lambda i}{\theta+2 \lambda i}
$$

and corresponding differential scattering phase

$$
\varphi_{\mathrm{LL}}(\theta)=\frac{4 \lambda}{\theta^{2}+4 \lambda^{2}} .
$$

TABLE I. The functions $j^{\text {sta }}, \quad\left[\left(e^{L}-e^{R}\right) / 2\right]$, and $\left[\left(k^{L}-k^{R}\right) / 2\right]$ over a wide range of values of $\beta_{L}$. The bounds [Eq. (3)] are always met.

\begin{tabular}{lccc}
\hline \hline$\beta_{L}$ & $\dot{j}^{\text {sta }}$ & {$\left[\left(e^{L}-e^{R}\right) / 2\right]$} & {$\left[\left(\kappa^{L}-\hbar^{R}\right) / 2\right]$} \\
\hline $10^{-5}$ & $2.5661 \times 10^{9}$ & $2.5701 \times 10^{9}$ & $2.5624 \times 10^{9}$ \\
$10^{-4}$ & $2.5450 \times 10^{7}$ & $2.5522 \times 10^{7}$ & $2.5386 \times 10^{7}$ \\
$10^{-3}$ & 250665.6 & 252117.9 & 249421.1 \\
$10^{-2}$ & 2424.9 & 2461.8 & 2396.4 \\
$10^{-1}$ & 22.0 & 23.3 & 21.1 \\
1 & 0.126 & 0.181 & 0.101 \\
\hline \hline
\end{tabular}

A uniform chemical potential $\mu$ is introduced, associated to the conserved charge $Q_{0}$ that counts the number of quasiparticles [with $h_{0}(\theta)=1$ ]. The energy current is chosen to be the current associated to the charge $H-\mu Q_{0}$,

$$
\dot{j}:=\dot{j}_{1}-\mu_{j_{0}} \quad \text { (LL model). }
$$

Below, we present some numerical results for several values of the coupling $\lambda$ and for $m=1$.

Current profiles obtained for $\lambda=3$ and various values of $\mu$ are displayed in Fig. 5. The main difference between the relativistic and nonrelativistic cases is the lack, in the latter, of any sharp light-cone effect. Nevertheless, at low temperatures $T_{L, R} \ll \mu$, Luttinger liquid physics emerges [73], including an emerging light cone due to the Fermi velocity. This can be seen in Fig. 5: a plateau forms whose height is again in agreement with the general CFT result Eq. (2). The plateau lies between nearly symmetric values $\xi / v_{F} \approx \pm 1$ fixed by the Fermi velocity $v_{F}$. Thermal occupation numbers $n^{L, R}(\theta)$ are very sharply supported between Fermi points $\pm \theta_{F}^{L, R}$, with $\theta^{L, R} \gtrsim \sqrt{2 \mu / m}$, and the Fermi velocity, which depends on $\xi$ very weakly, is the effective velocity $v^{\text {eff }}\left(\theta_{F}^{R}\right)$ associated to the lowest temperature $\left(T_{R}<T_{L}\right.$ in the present example). In agreement with general CFT results [26,27], a light cone thus builds up (despite the model having no intrinsic maximal velocity), and the full state is, in fact, homogeneous between the Fermi velocities.

In the LL model the coupling constant may take any values between 0 and $\infty$ and the limits $\lambda \rightarrow 0$ and $\lambda \rightarrow \infty$ are of particular interest.

For $\lambda \rightarrow 0$, the differential scattering phase Eq. (44) becomes heavily peaked around $\theta=0$. Formally, $\lim _{\lambda \rightarrow 0} \varphi_{\mathrm{LL}}(\theta)=2 \pi \delta(\theta)$. The resulting TBA equations,

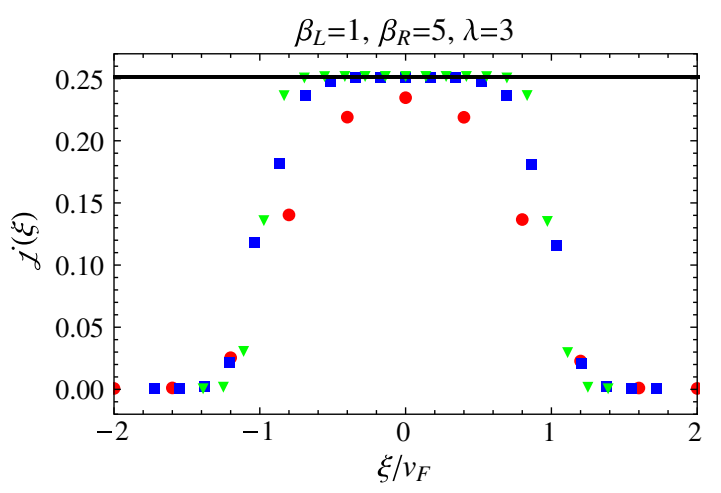

FIG. 5. Energy current in the Lieb-Liniger model for low temperatures $\lambda=3$ and chemical potentials $\mu=3$ (circles), $\mu=6$ (squares), and $\mu=10$ (triangles). The CFT value $\frac{\pi}{12}\left(1-\frac{1}{25}\right)$ (bold horizontal line) is reached for high values of $\mu$. By plotting the currents against $\xi / v_{F}$, we observe the collapse of the various curves, which becomes better as $\mu$ increases. The regions where plateaus emerge are roughly $\xi / v_{F} \in[-1,1]$ with $v_{F} \approx 2.5,2.9,3.6$. 


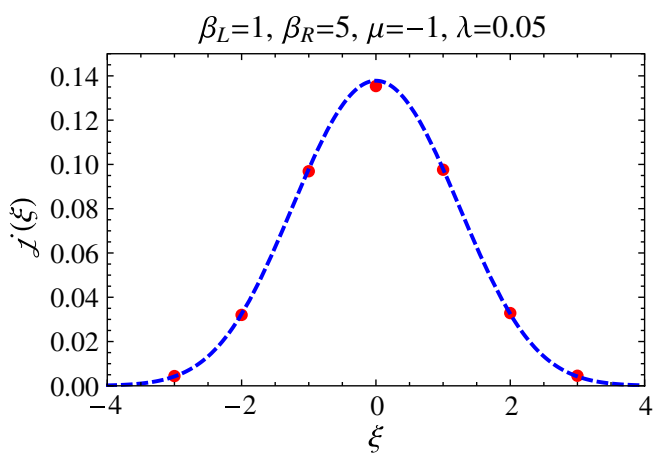

FIG. 6. Energy current in the Lieb-Liniger model for low temperatures, small coupling, and negative chemical potential (circles). The dashed curve represents the current [Eq. (46)] for the same temperatures and chemical potential.

with this differential scattering phase, admit no solution for the pseudoenergy if $\mu>0$, but for $\mu<0$, they can be solved exactly and reproduce the free boson solution (for which $\mu>0$ would make no physical sense). In particular, the energy current takes the free boson form,

$\lim _{\lambda \rightarrow 0} \dot{j}(\xi)=\frac{1}{\beta_{R}^{2}} \int_{\alpha_{R}}^{\infty} d \theta \frac{\theta}{e^{\theta}-1}-\frac{1}{\beta_{L}^{2}} \int_{\alpha_{L}}^{\infty} d \theta \frac{\theta}{e^{\theta}-1}$,

where $\alpha_{L, R}=\beta_{L, R}\left[\left(\xi^{2} / 2\right)-\mu\right]$. In Fig. 6, we compare numerical values for $\lambda=0.05$ and $\mu=-1$ to this analytical expression. The agreement is very good, confirming that a free boson theory is smoothly recovered in this limit. With $\mu>0$, as $\lambda$ becomes small, the TBA equations gradually break down. How this occurs is subtle, and will be discussed in Ref. [74].

The qualitative change in behavior of the TBA solutions as $\lambda \rightarrow 0$ is related to the two distinct regimes observed at small values of $\lambda$ [75]. Consider the dimensionless coupling $\gamma:=2 \mathrm{m \lambda} / q_{0}$ (where we recall that $q_{0}$ is the gas density, which may be taken in the initial baths, for instance) and the reduced temperature $\tau:=2 m T / q_{0}^{2}$. The "decoherent regime," with large phase and density fluctuations, occurs for $\gamma \lesssim \min \left(\tau^{2}, \sqrt{\tau}\right)$. In this regime, ideal Bose gas physics is recovered, and we indeed verify that the inequality is satisfied in the parameter space where good agreement with Eq. (46) is observed (small $\lambda$, negative $\mu$ ). On the other hand, the "Gross-Pitaevskii regime" occurs for $\tau^{2} \lesssim \gamma \lesssim 1$, a quasicondensate with large phase fluctuations but suppressed density fluctuations. It is such quasicondensate physics that strongly affects TBA solutions as $\lambda \rightarrow 0$ with $\mu>0$.

The other interesting limit is $\lim _{\lambda \rightarrow \infty} \varphi_{\mathrm{LL}}(\theta)=0$. In this case, we can also find an analytical expression for the current:

$\lim _{\lambda \rightarrow \infty} j(\xi)=\frac{1}{\beta_{R}^{2}} \int_{\alpha_{R}}^{\infty} d \theta \frac{\theta}{e^{\theta}+1}-\frac{1}{\beta_{L}^{2}} \int_{\alpha_{L}}^{\infty} d \theta \frac{\theta}{e^{\theta}+1}$.
This corresponds to a free Fermion, in agreement with the expected Tonks-Girardeau physics occurring in the regime $\gamma \gtrsim \max (1, \sqrt{\tau})$ [75]. For $\xi \approx 0$ and $\mu \beta_{L, R} \gg 1$, it is easy to show that the integral above gives $\frac{\pi}{12}\left(\beta_{L}^{-2}-\beta_{R}^{-2}\right)$, so that we recover the CFT result for the current with $c=1$ (Dirac fermion). Figure 7 shows a comparison between numerical values of the current for $\lambda=50$ and the formula above.

Let us now consider the particle current. Naturally, in the LL model, equilibrium states at higher temperatures have lower particle densities. Therefore, although the energy current flows from the left to the right in the present setup (with $T_{L}>T_{R}$ ), the initial particle density imbalance would naively suggest a particle flow from the right (higher density) to the left (lower density). The opposite occurs: Figure 9 shows that the particle current is positive, hence, flows form the left to the right. This means that the fluid flow produced by the temperature difference drags particles with enough force to counteract the particle imbalance and bring particles towards the higher-density bath. The fact that heat carries particles along its motion is a thermoelectric effect. It has been experimentally demonstrated in a quasi-two-dimensional fermionic cold atoms channel [3], and theoretically shown in CFT in dimensions higher than 1 [17]. It is nontrivial in integrable models, as the large amount of conservation laws allows for independent currents to coexist, and our result gives the first theoretical prediction of this effect in the integrable one-dimensional Bose gas.

An additional consequence of the thermoelectric effect is that the particle density $q_{0}(\xi)$ shows particle accumulation around $v_{F}$ and depletion around $-v_{F}$ (see Fig. 8). For instance, the start of the dip can be explained by the fact that, in any local spacial region originally in the left reservoir, the first particles to start moving towards the

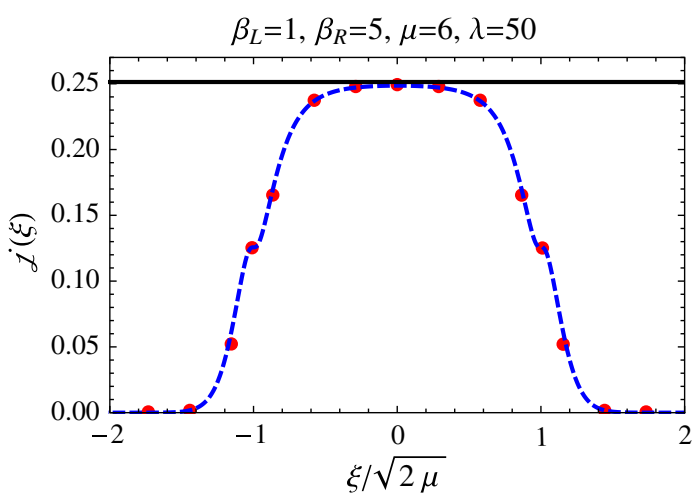

FIG. 7. Energy current in the Lieb-Liniger model for low temperatures, large coupling, and chemical potential $\mu=6$ (circles). Local stationary points occur at $\alpha_{L, R}=0$, that is, $\xi= \pm \sqrt{2 \mu}= \pm 3.46$ (the Fermi velocity). The dashed curve represents the current [Eq. (47)] for the same temperatures and chemical potential, whose profile is not dissimilar to the plots shown in Fig. 5. As before, the bold horizontal line is the CFT value $\frac{\pi}{12}\left(1-\frac{1}{25}\right)$. The agreement is extremely good. 


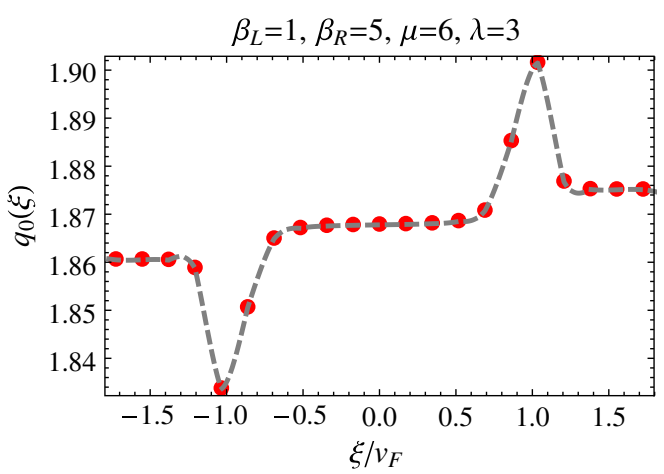

FIG. 8. A characteristic profile of the Lieb-Liniger particle density for $T_{L, R} \ll \mu, \lambda=3$, and $\mu=6$. The local maxima and minima are located around $\xi= \pm v_{F}$. The dashed curve is an interpolation.

right are those on the right of the region, escaping and thus depleting it. Since time evolution at fixed position is obtained by scanning Fig. 8 from left to right, this explains the initial dip on the left. This depleting effect continues as long as the outgoing current on the right of the region is higher then the incoming current on its left-that is, until the region lies in the steady state. However, as time goes on, the effective local temperature decreases, and this tends to increase the particle density. This effect eventually overtakes the depleting effect, accounting for the rebounce to the higher steady-state value. The behavior of the current $\dot{z}_{0}$ in Fig. 9 is then a consequence of the continuity equation $\xi \partial_{\xi} q_{0}=\partial_{\xi} \dot{\alpha_{0}}$.

This is a nonuniversal effect, not present in the density $q_{1}(\xi)-\mu q_{0}(\xi)$ controlled by low-energy processes, where the physics of chiral separation dominates and monotonic transition regions occur.

\section{General features}

The form of the nonequilibrium occupation number indicates that quasiparticles are thermalized according to

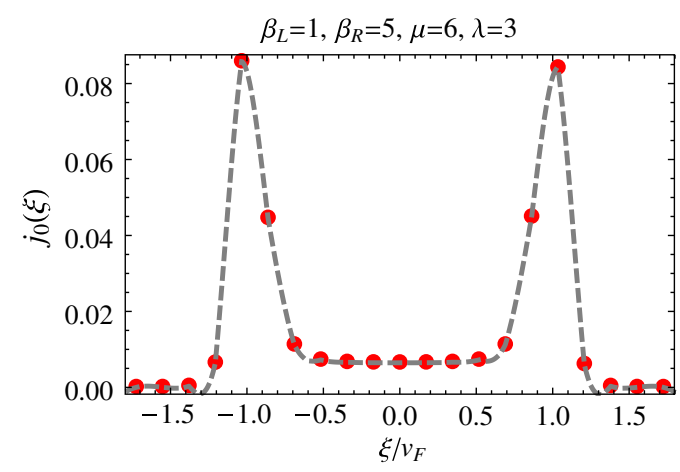

FIG. 9. A characteristic profile of the Lieb-Liniger particle current for $T_{L, R} \ll \mu, \lambda=3$, and $\mu=6$. The local maxima and minima are located around $\xi= \pm v_{F}$. The dashed curve is an interpolation.

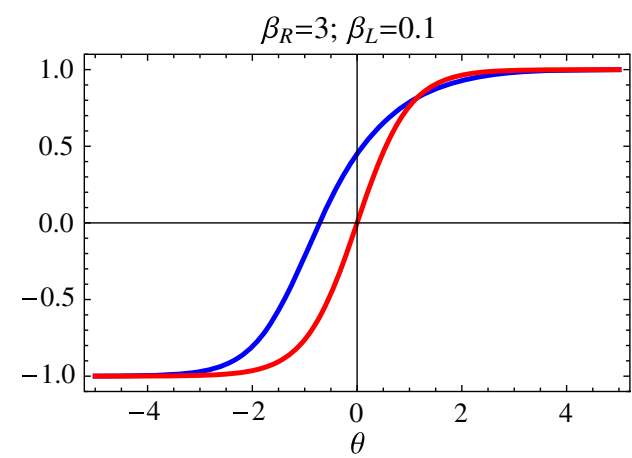

FIG. 10. Effective velocity in the sinh-Gordon model for $\xi=0$. Displayed are the effective velocity $v^{\text {eff }}(\theta)$ (blue line) and the bare relativistic velocity $\tanh \theta$ (red line).

the initial GGEs, in a way that depends on the rapidity. It connects with the picture, proposed in Refs. [26,47], according to which, in the steady state $(\xi=0)$, quasiparticles traveling towards the right (left) are thermalized according to the left (right) reservoir. However, in the present solution, what determines the traveling direction is the effective velocity in the steady state: quasiparticles with positive (negative) dressed velocities, reaching the point $x=0$ at large times, will have traveled mostly towards the right (left) (after a relatively small transient). In the sinhGordon model with $T_{L}>T_{R}$, the effective velocity behaves as in Fig. 10. We observe that it is greater than the bare velocity $\tanh \theta$ for small or negative rapidities, and smaller for large positive rapidities. This is in agreement with the intuition according to which the quasiparticles are effectively carried by the flow, which transports them towards the right, for small enough rapidities, but slowed down by dominant "friction" effects of thermal fluctuations at large rapidities. A similar effect occur in the Lieb-Liniger model.

The generalized hydrodynamic result differs from previous proposals in interacting integrable models [47-49] (while all results agree in noninteracting cases). The original proposal [47] was later shown [44] to break the second inequality in Eq. (3), while the second proposal [48], based on similar ideas, gave slight disagreements with numerical simulations. The conjecture [49], which corresponds to taking $\theta_{\star}=0$ in our framework, seems to give good agreement with numerical simulations. This may be due to the fact that taking $\theta_{\star}=0$, gives very small errors in wide temperature ranges, of the order of $0.5 \%-1 \%$ (we confirm this numerically).

\section{CONCLUSIONS}

In this paper, we develop a hydrodynamic theory for infinitely many conservation laws and apply it to the study of heat flows in experimentally relevant integrable models. It would be interesting to study further the nonequilibrium physics of the Lieb-Liniger model, including the effects of 
the Gross-Pitaevskii quasicondensate and transport between different regimes. The emerging physical picture and solution we give can be applied to any Bethe ansatz integrable model, where infinitely many conservation laws exist and a quasiparticle description is available. This includes quantum chains (see Ref. [64]), as continuity of space on which the microscopic theory lies is not needed for emerging hydrodynamics. It also includes relativistic models with nondiagonal scattering, such as the sineGordon model, where, for instance, our TBA construction may be generalized along the lines of the famous approach of Destri and De Vega [76,77]. Of course, the hydrodynamic ideas do not require a quasiparticle description, and it might be possible to develop generalized hydrodynamics using a variety of techniques from integrability. We note that it is remarkable that independent quasiparticle mode thermalization agrees, in integrable models, with local entropy maximization. The dynamical equations derived can be used to describe more general situations in ultracold gases, such as the release from a trap (see, e.g., Ref. [78]). This new theory and its extensions, including viscosity effects and forcing, should also allow for efficient studies of integrability breaking and related problems in any dimensionality, as well as for exact descriptions of dynamics in smooth trapping potentials [4] at arbitrary coupling strength.

\section{ACKNOWLEDGMENTS}

We thank Denis Bernard, M. Joe Bhaseen, JeanSébastien Caux, Fabian Essler, Mauricio Fagotti, and Eric Lutz for discussions, and especially Bruno Bertini and Lorenzo Piroli for sharing their preliminary ideas and Pasquale Calabrese for encouraging us to pursue this research. O. A. C.-A. and B.D. are grateful to SISSA, Trieste, Italy, for support during a visit where this work started. T. Y. thanks the Takenaka Scholarship Foundation for financial support. Open access for this article was funded by King's College London.

Note added.-Recently, similar dynamical equations as those derived here were independently obtained in the integrable $X X Z$ Heisenberg chain by assuming, in addition to local entropy maximization, an underlying kinetic theory [64]. Solutions to these equations of the same type as those considered here were constructed and confirmed by numerical simulations.

\section{APPENDIX A: CURRENT GENERATORS}

Let $\langle\cdots\rangle_{\beta}$ be the state given by Eq. (4), and $\langle a(x) b(y)\rangle^{c}:=\langle a(x) b(y)\rangle_{\underline{\beta}}-\langle a(x)\rangle_{\underline{\beta}}\langle b(y)\rangle_{\underline{\beta}}$ the connected correlation functions. These are time independent and functions of the difference $x-y$ only. Let us assume that connected correlation functions of conserved densities and currents vanish faster than the inverse distance $|x-y|$. Then,

$$
\begin{aligned}
\int d x\left\langle q_{m}(x) j_{n}(0)\right\rangle^{c} & =\int d x\left\langle j_{n}(0) q_{m}(x)\right\rangle^{c} \\
& =\int d x\left\langle j_{n}(x) q_{m}(0)\right\rangle^{c} \\
& =-\int d x x\left\langle\partial_{x} j_{n}(x) q_{m}(0)\right\rangle^{c} \\
& =\int d x x\left\langle\partial_{t} q_{n}(x) q_{m}(0)\right\rangle^{c} \\
& =-\int d x x\left\langle q_{n}(x) \partial_{t} q_{m}(0)\right\rangle^{c} \\
& =\left.\int d x x\left\langle q_{n}(x) \partial_{y} j_{m}(y)\right\rangle^{c}\right|_{y=0} \\
& =-\int d x x \partial_{x}\left\langle q_{n}(x) j_{m}(0)\right\rangle^{c} \\
& =\int d x\left\langle q_{n}(x) j_{m}(0)\right\rangle^{c} .
\end{aligned}
$$

In the first line, we use the fact that $\int d x q_{m}(x)$ is a conserved quantity and thus commutes with the density matrix $\rho$; in the second line, we use space translation invariance; in the third, integration by parts and the fastenough vanishing of correlation functions; in the fourth, current conservation; in the fifth, time-translation invariance; in the sixth, current conservation; in the seventh, space-translation invariance; and in the eighth, integration by parts. Therefore,

$$
\frac{\partial}{\partial \beta_{m}} \dot{z}_{n}=\frac{\partial}{\partial \beta_{n}} \dot{z}_{m}
$$

and, thus,

$$
\dot{\mathcal{L}}_{m}=\frac{\partial}{\partial \beta_{m}} g_{\underline{\beta}},
$$

showing Eq. (6).

In the TBA context, we note that expressions (22) show the existence of appropriate free energies $f_{w}$ and $g_{w}$ generating densities and currents, respectively, as in Eq. (6). Indeed, they may be rewritten as

$$
\begin{aligned}
& q_{i}=\int d \theta h_{i}(\theta) \frac{\delta f_{w}}{\delta w(\theta)}, \\
& f_{w}=-\int \frac{d p(\alpha)}{2 \pi} \log \left(1+e^{-\epsilon_{w}(\alpha)}\right)
\end{aligned}
$$

and

$$
\begin{aligned}
\dot{z}_{i} & =\int d \theta h_{i}(\theta) \frac{\delta g_{w}}{\delta w(\theta)}, \\
g_{w} & =-\int \frac{d E(\alpha)}{2 \pi} \log \left(1+e^{-\epsilon_{w}(\alpha)}\right) .
\end{aligned}
$$


It then follows that that functional $w(\theta)$ derivatives of these free energies give the quasiparticle and current densities, $\rho_{p}(\theta)=\delta f_{w} / \delta w(\theta)$ and $\rho_{c}(\theta)=\delta g_{w} / \delta w(\theta)$.

\section{APPENDIX B: EMERGENCE OF GENERALIZED HYDRODYDNAMICS}

The only principle at the basis of hydrodynamics, and of the derivation we provide, is that of the emergence of local generalized thermalization (local entropy maximization). Technically, this is the assumption that averages of local quantities $\langle\mathcal{O}(x, t)\rangle$ tend uniformly enough, at large $x$ and $t$, to averages evaluated in GGEs (infinite-volume, maximalentropy states, under conditions on infinitely many conservation laws), with space-time-dependent potentials. This assumption is sufficient to derive the explicit dynamics for all single-point averages of local conserved densities and currents: no ad hoc kinetic principle is needed.

In the case of infinitely many conservation laws, one delicate point is the consideration of quasilocal densities and currents, which are involved in generalized thermalization. Such a quantity is not supported on a finite region, but on an extended region, with a weight (as measured by, for instance, the overlap with any other local observable) that decays away from a point. However, since hydrodynamics is concerned with large-scale space-time regions (the fluid cells), it is natural to consider them on the same footing. This is implicitly done in the derivation we present in this paper by assuming a completeness property of conservation laws.

Another delicate point concerns the definition of GGEs. In finite systems, such states depend on the boundary conditions imposed, and, in general, these boundary conditions may still have an effect in the infinite-volume limit. For instance, walls simply preclude any nonzero potential associated to the momentum operator, as they break translation invariance. Nevertheless, given a set of allowed conserved charges, at large volumes, boundary conditions have little effect on local averages of conserved currents and densities (as they do not affect specific free energies). Further, periodic boundary conditions, at the basis of the TBA formalism, appear to provide the maximal set of conserved charges. It is, in fact, possible to construct GGEs directly in infinite volumes [21]. We expect local thermalization, and the full set of available conserved charges, to be correctly described by such constructions, and we expect these to agree with the TBA formalism we use here.

We finally mention that the classical hard-rod problem, proven to give rise to a form of hydrodynamics [37,53], has strong connections with the integrable systems we investigate here, which we will investigate in a future work.

\section{APPENDIX C: MANY-PARTICLE SPECTRUM}

The theory we develop here is directly applicable to any integrable model whose two-particle scattering is diagonal in the internal space. Let the spectrum be composed of $\ell$ particles, of masses $m_{a}, a=1, \ldots, \ell$, and assume that their scattering is diagonal. In this case, the TBA equations can still be applied [61,79]: the differential scattering phase is replaced by a matrix of functions $\varphi_{a b}(\theta-\gamma)$, and the oneparticle eigenvalue of $Q_{i}$ is denoted by $h_{i}(\theta ; a)$. The solution $\underline{q}(\xi), \underline{j}(\xi)$ of the generalized hydrodynamic problem is

$$
\begin{aligned}
& q_{i}(\xi)=\sum_{a} \int \frac{d p(\theta ; a)}{2 \pi} n(\theta ; a) h_{i}^{\mathrm{dr}}(\theta ; a), \\
& \dot{\mathcal{L}}_{i}(\xi)=\sum_{a} \int \frac{d E(\theta ; a)}{2 \pi} n(\theta ; a) h_{i}^{\mathrm{dr}}(\theta ; a),
\end{aligned}
$$

where $p(\theta ; a)=m_{a} \sinh \theta, E(\theta ; a)=m_{a} \cosh \theta$, and

$h_{i}^{\mathrm{dr}}(\theta ; a)=h_{i}(\theta ; a)+\int \frac{d \gamma}{2 \pi} \sum_{b} \varphi_{a, b}(\theta-\gamma) n(\gamma ; b) h_{i}^{\mathrm{dr}}(\gamma ; b)$.

The nonequilibrium occupation number $n(\theta ; a)$ is given by the discontinuous function

$n(\theta ; a)=n^{L}(\theta ; a) \Theta\left(\theta-\theta_{\star}(a)\right)+n^{R}(\theta ; a) \Theta\left(\theta_{\star}(a)-\theta\right)$,

where each particle $a$ is associated to a different discontinuity at position $\theta_{\star}(a)$. These positions are selfconsistently determined by the zeros of the dressed, boosted momenta of particles $a$; with $p_{\xi}(\theta ; a):=$ $m_{a} \sinh (\theta-\eta)$ (relativistic) or $m_{a} \theta$ (nonrelativistic):

$$
p_{\xi}^{\mathrm{dr}}\left(\theta_{\star}(a) ; a\right)=0, \quad a=1, \ldots, \ell .
$$

Again, the thermal occupation numbers $n^{L, R}(\theta ; a)$ entering Eq. (C3) guarantee that the asymptotic conditions on $\xi$ correctly represent the asymptotic baths, as per Eq. (14). They are obtained using the TBA equations in terms of the pseudoenergies $\epsilon_{w}(\theta ; a)[61,79]$,

$$
\begin{aligned}
n^{L, R}(\theta ; a)= & \frac{1}{1+\exp \left[\epsilon_{w^{L, R}}(\theta ; a)\right]}, \\
\epsilon_{w}(\theta ; a)= & w(\theta ; a)- \\
& -\int \frac{d \gamma}{2 \pi} \sum_{b} \varphi_{a, b}(\theta-\gamma) \log \left(1+e^{-\epsilon_{w}(\gamma ; b)}\right) .
\end{aligned}
$$

Here, $w^{L, R}(\theta ; a)=\sum_{i} \beta_{i}^{L, R} h_{i}(\theta ; a)$ are the one-particle eigenvalues of the charge $\sum_{i} \beta_{i}^{L, R} Q_{i}$ characterizing the GGE of the left and right asymptotic reservoirs. 


\section{APPENDIX D: CURRENT AVERAGES}

An alternative proof of Eq. (22) may be given using the technology of integrable systems, which has the advantage of generalizing to flows generated by any conserved charge instead of just the Hamiltonian. For completeness, we present here the main arguments. The idea is to prove expression (22) for current averages $\dot{z}_{i}$ given the expression for density averages $q_{i}$. This is akin to extending the LeClair-Mussardo (LM) formula [80] so that it incorporates the infinite number of conserved charges, and applying it to the current with the aid of form factors (FFs). We use the notation introduced in Ref. [81]. Following the derivation in Ref. [81], we generalize the LM formula for a one-point function of a generic local operator $\mathcal{O}(x, t)$ :

$$
\langle\mathcal{O}(x, t)\rangle=\sum_{\ell=0}^{\infty} \frac{1}{\ell !}\left(\prod_{k=1}^{\ell} \frac{d \theta_{k}}{2 \pi} n\left(\theta_{k}\right)\right)\langle\overleftarrow{\theta}|\mathcal{O}(0)| \vec{\theta}\rangle_{c}
$$

where $|\vec{\theta}\rangle=\left|\theta_{1}, \ldots, \theta_{\ell}\right\rangle$ (and $\langle\overleftarrow{\theta}|=\left\langle\theta_{\ell}, \ldots, \theta_{1}\right|$ is its Hermitian conjugate) and diagonal matrix elements (DMEs) in the sum are connected (the meaning of being "connected" is described below). Here, $n(\theta)$ is the same occupation number as that involved in Eqs. (20), (21), and (22). It is then immediate to see that an expression for the density average $q_{i}$ with the one-particle eigenvalue $h_{i}(\theta)$ proved by Saleur [82] is modified to

$$
\begin{aligned}
q_{i}= & m \sum_{\ell=0}^{\infty}\left(\prod_{k=1}^{\ell} \frac{d \theta_{k}}{2 \pi} n\left(\theta_{k}\right)\right) \\
& \times \varphi\left(\theta_{12}\right) \ldots \varphi\left(\theta_{\ell-1, \ell}\right) h_{i}\left(\theta_{1}\right) \cosh \theta_{\ell},
\end{aligned}
$$

where $\varphi\left(\theta_{i j}\right)=\varphi\left(\theta_{i}-\theta_{j}\right)$. Observe that this is indeed in agreement with the expression in Eq. (22). The expression (D2) can be derived using the DMEs of $q_{i}(x, t)$ given by

$$
\begin{aligned}
\left\langle\overleftarrow{\theta}\left|q_{i}\right| \vec{\theta}\right\rangle_{c}= & m \varphi\left(\theta_{12}\right) \varphi\left(\theta_{23}\right) \ldots \varphi\left(\theta_{\ell-1, \ell}\right) \\
& \times h_{i}\left(\theta_{1}\right) \cosh \theta_{\ell}+\text { permutations. }
\end{aligned}
$$

Similarly, once we evaluate DMEs for the current $j_{i}(x, t)$, we can construct its average $\dot{\mathcal{L}}_{i}$. The expression in Eq. (22), that we want to show, will then follow if the DMEs of the currents are obtained from those of the densities by the replacement of $\cosh \theta_{n}$ with $\sinh \theta_{n}$ :

$$
\begin{aligned}
\left\langle\overleftarrow{\theta}\left|j_{i}\right| \vec{\theta}\right\rangle_{c}= & m \varphi\left(\theta_{12}\right) \varphi\left(\theta_{23}\right) \cdots \varphi\left(\theta_{\ell-1, \ell}\right) \\
& \times h_{i}\left(\theta_{1}\right) \sinh \theta_{\ell}+\text { permutations. }
\end{aligned}
$$

Before embarking upon showing it, we elaborate on the definitions of connected and symmetric DMEs. Formally, they are given by, respectively [83],

$$
\begin{aligned}
\langle\overleftarrow{\theta}|\mathcal{O}| \vec{\theta}\rangle_{c} & :=F_{2 \ell}^{c}(\mathcal{O} ; \vec{\theta}) \\
& :=\mathrm{FP}_{\delta_{k} \rightarrow 0} F_{2 \ell}(\mathcal{O} ; \vec{\theta}+i \pi+\vec{\delta}, \overleftarrow{\theta}), \\
\langle\overleftarrow{\theta}|\mathcal{O}| \vec{\theta}\rangle_{s} & :=F_{2 \ell}^{s}(\mathcal{O} ; \vec{\theta}) \\
& :=\lim _{\delta \rightarrow 0} F_{2 \ell}(\mathcal{O} ; \vec{\theta}+i \pi+\delta, \overleftarrow{\theta}),
\end{aligned}
$$

where FP means "finite part" [81], $\vec{\delta}=\left(\delta_{1}, \ldots, \delta_{\ell}\right)$, and the FF $F_{\ell}(\mathcal{O} ; \vec{\theta})$ is defined by

$$
F_{\ell}(\mathcal{O} ; \vec{\theta})=\langle\operatorname{vac}|\mathcal{O}(0)| \vec{\theta}\rangle \text {. }
$$

Notice that with a limit such as in Eq. (D5), where the parameters $\delta_{k}$ differ in each component, different orders of limits lead to different results which may be singular; this is because when $\delta_{k} \rightarrow 0$, the FF [Eq. (D5)] becomes singular due to kinematic poles. It is in order to circumvent this ambiguity that one defines connected and symmetric FFs. The connected FF is a finite part, which simply prescribes to set to zero terms with singularities in $\delta_{k}$ [81], whereas the symmetric FF is defined by sending all parameters to zero simultaneously.

It was pointed out in Ref. [83] that any multiparticle symmetric FF can be written solely in terms of the connected FFs. For instance, for a two-particle state, the connected FF $F_{4}^{c}\left(\mathcal{O} ; \theta_{1}, \theta_{2}\right)$ and the symmetric $\mathrm{FF}$ $F_{4}^{s}\left(\mathcal{O} ; \theta_{1}, \theta_{2}\right)$ satisfy

$$
\begin{aligned}
F_{4}^{c}\left(\mathcal{O} ; \theta_{1}, \theta_{2}\right)= & F_{4}^{s}\left(\mathcal{O} ; \theta_{1}, \theta_{2}\right)-\varphi\left(\theta_{12}\right) F_{2}\left(\mathcal{O} ; \theta_{1}\right) \\
& -\varphi\left(\theta_{21}\right) F_{2}\left(\mathcal{O} ; \theta_{2}\right),
\end{aligned}
$$

where $F_{2}(\mathcal{O} ; \theta)=F_{2}^{c}(\mathcal{O} ; \theta)=F_{2}^{s}(\mathcal{O} ; \theta)$ (in the case of a single parameter $\delta_{1}$, there is no singularity, hence, no ambiguity). Applying this relation to $j_{i}$, we have

$$
\begin{aligned}
F_{4}^{c}\left(j_{i} ; \theta_{1}, \theta_{2}\right)= & F_{4}^{s}\left(j_{i} ; \theta_{1}, \theta_{2}\right)-\varphi\left(\theta_{12}\right) F_{2}\left(j_{i} ; \theta_{1}\right) \\
& -\varphi\left(\theta_{21}\right) F_{2}\left(j_{i} ; \theta_{2}\right) .
\end{aligned}
$$

This can be expressed in terms of FFs of the density $q_{i}$ thanks to the conservation law $\partial_{t} q_{i}+\partial_{x} j_{i}=0$, which entails

$$
F_{2 \ell}^{s}\left(j_{i} ; \vec{\theta}\right)=\frac{\sum_{k=1}^{\ell} \sinh \theta_{k}}{\sum_{k=1}^{\ell} \cosh \theta_{k}} F_{2 \ell}^{s}\left(q_{i} ; \vec{\theta}\right)
$$

Hence, putting Eq. (D10) into Eq. (D9) yields

$F_{4}^{c}\left(j_{i} ; \theta_{1}, \theta_{2}\right)=m \varphi\left(\theta_{12}\right) h_{i}\left(\theta_{1}\right) \sinh \theta_{2}+\left\{\theta_{1} \leftrightarrow \theta_{2}\right\}$,

which is consistent with Eq. (D4). It is readily seen that for multiparticle states, similar arguments hold, and, thus, we 
obtain Eq. (D4). Finally, the generalized LM formula for the current gives

$$
\begin{aligned}
\dot{z}_{i}= & m \sum_{\ell=0}^{\infty}\left(\prod_{k=1}^{\ell} \frac{d \theta_{k}}{2 \pi} n\left(\theta_{k}\right)\right) \\
& \times \varphi\left(\theta_{12}\right) \cdots \varphi\left(\theta_{\ell-1, \ell}\right) h_{i}\left(\theta_{1}\right) \sinh \theta_{\ell} .
\end{aligned}
$$

This exactly coincides with Eq. (22). Similar arguments give rise to current averages associated to flows $i\left[Q_{k}, q_{i}\right]+$ $\partial_{x} j_{i}^{(k)}=0$ with respect to any local conserved quantity $Q_{k}$ (with odd spin):

$$
\dot{L}_{i}^{(k)}=\int \frac{d h_{k}(\theta)}{2 \pi} n(\theta) h_{i}^{\mathrm{dr}}(\theta)
$$

A full derivation will be given in Ref. [74].

[1] J. Eisert, M. Friesdorf, and C. Gogolin, Quantum Many-Body Systems Out of Equilibrium, Nat. Phys. 11, 124 (2015).

[2] S. Jezouin, F. D. Parmentier, A. Anthore, U. Gennser, A. Cavanna, Y. Jin, and F. Pierre, Quantum Limit of Heat Flow across a Single Electronic Channel, Science 342, 601 (2013).

[3] J.-P. Brantut et al., A Thermoelectric Heat Engine with Ultracold Atoms, Sci. Rep. 342, 713 (2013).

[4] T. Kinoshita, T. Wenger, and D. S. Weiss, A Quantum Newton's Cradle, Nature (London) 440, 900 (2006).

[5] T. Langen, S. Erne, R. Geiger, B. Rauer, T. Schweigler, M. Kuhnert, W. Rohringer, I. E. Mazets, T. Gasenzer, and J. Schmiedmayer, Experimental Observation of a Generalized Gibbs Ensemble, Science 348, 207 (2015).

[6] M. Cheneau, P. Barmettler, D. Poletti, M. Endres, P. Schauß, T. Fukuhara, C. Gross, I. Bloch, C. Kollath, and S. Kuhr, Light-Cone-like Spreading of Correlations in a Quantum Many-Body System, Nature (London) 481, 484 (2012).

[7] D. Ruelle, Natural Nonequilibrium States in Quantum Statistical Mechanics, J. Stat. Phys. 98, 57 (2000).

[8] P. Nozieres and D. Pines, The Theory of Quantum Liquids (Benjamin, New York, 1966).

[9] S. Jeon and L. G. Yaffe, From Quantum Field Theory to Hydrodynamics: Transport Coefficients and Effective Kinetic Theory, Phys. Rev. D 53, 5799 (1996).

[10] A. G. Abanov, in Applications of Random Matrices in Physics, edited by E. Brézin, V. Kazakov, D. Serban, P. Wiegmann, and A. Zabrodin, NATO Science Series II: Mathematics, Physics and Chemistry, Vol. 221 (Springer, Dordrecht, 2006), pp. 139-161.

[11] E. Bettelheim, A. G. Abanov, and P. Wiegmann, Nonlinear Quantum Shock Waves in Fractional Quantum Hall Edge States, Phys. Rev. Lett. 97, 246401 (2006).

[12] A. Bressan, Modelling and Optimisation of Flows on Networks, Lect. Notes Math. 2062, 157 (2013).
[13] J. Bhaseen, B. Doyon, A. Lucas, and K. Schalm, Far from Equilibrium Energy Flow in Quantum Critical Systems, Nat. Phys. 11, 509 (2015).

[14] H.-C. Chang, A. Karch, and A. Yarom, An Ansatz for One Dimensional Steady State Configurations, J. Stat. Mech. (2014) P06018.

[15] D. Bernard and B. Doyon, A Hydrodynamic Approach to Non-Equilibrium Conformal Field Theories, J. Stat. Mech. (2016) 033104.

[16] R. Pourhasan, Non-Equilibrium Steady State in the Hydro Regime, J. High Energy Phys. 02 (2016) 005.

[17] A. Lucas, K. Schalm, B. Doyon, and M. J. Bhaseen, Shock Waves, Rarefaction Waves and Nonequilibrium Steady States in Quantum Critical Systems, Phys. Rev. D 94, 025004 (2016).

[18] M. Spillane and C. P. Herzog, Relativistic Hydrodynamics and Non-Equilibrium Steady States, arXiv:1512.09071.

[19] M. Rigol, V. Dunjko, V. Yurovsky, and M. Olshanii, Relaxation in a Completely Integrable Many-Body Quantum System: An Ab Initio Study of the Dynamics of the Highly Excited States of 1D Lattice Hard-Core Bosons, Phys. Rev. Lett. 98, 050405 (2007).

[20] E. Ilievski, J. De Nardis, B. Wouters, J.-S. Caux, F. H. L. Essler, and T. Prosen, Complete Generalized Gibbs Ensemble in an Interacting Theory, Phys. Rev. Lett. 115, 157201 (2015).

[21] B. Doyon, Thermalization and Pseudo-Locality in Extended Quantum Systems, arXiv:1512.03713.

[22] F. Essler and M. Fagotti, Quench Dynamics and Relaxation in Isolated Integrable Quantum Spin Chains, J. Stat. Mech. (2016) 064002.

[23] T. Prosen, Open XXZ Spin Chain: NonEquilibrium Steady State and a Strict Bound on Ballistic Transport, Phys. Rev. Lett. 106, 217206 (2011).

[24] T. Prosen and E. Ilievski, Families of Quasilocal Conservation Laws and Quantum Spin Transport, Phys. Rev. Lett. 111, 057203 (2013).

[25] E. Ilievski, M. Medenjak, T. Prosen, and L. Zadnik, Quasilocal Charges in Integrable Lattice Systems, J. Stat. Mech. (2016) 064008.

[26] D. Bernard and B. Doyon, Energy Flow in Non-equilibrium Conformal Field Theory, J. Phys. A 45, 362001 (2012).

[27] D. Bernard and B. Doyon, Non-Equilibrium Steady-States in Conformal Field Theory, Ann. Inst. Henri Poincaré 16, 113 (2015).

[28] D. Bernard and B. Doyon, Conformal Field Theory Out of Equilibrium: A Review, J. Stat. Mech. (2016) 064005.

[29] M. Moeckel and S. Kehrein, Interaction Quench in the Hubbard Model, Phys. Rev. Lett. 100, 175702 (2008).

[30] B. Bertini, F. H. L. Essler, S. Groha, and N. J. Robinson, Prethermalization and Thermalization in Models with Weak Integrability Breaking, Phys. Rev. Lett. 115, 180601 (2015).

[31] L. E. Reichl, The Search for a Quantum KAM Theorem, Found. Phys. 17, 689 (1987).

[32] G. P. Brandino, J.-S. Caux, and R. M. Konik, Glimmers of a Quantum KAM Theorem: Insights from Quantum Quenches in One-Dimensional Bose Gases, Phys. Rev. X 5, 041043 (2015).

[33] P. Glansdorff and I. Prigogine, Thermodynamic Theory of Structure, Stability, and Fluctuations (Wiley-Interscience, London, 1971). 
[34] R. Balescu, Equilibrium and Non-Equilibrium Statistical Mechanics (John Wiley and Sons, New York, 1975).

[35] J. Keizer, Statistical Thermodynamics of Nonequilibrium Processes (Springer-Verlag, New York, 1987).

[36] F. Schloegl, Probability and Heat: Fundamentals of Thermostatistics (Friedrig Vieweg und Sohn, Brausnchweig, 1989).

[37] H. Spohn, Large Scale Dynamics of Interacting Particles (Springer-Verlag, Berlin, 1991).

[38] E. H. Lieb and W. Liniger, Exact Analysis of an Interacting Bose Gas. I. The General Solution and the Ground State, Phys. Rev. 130, 1605 (1963).

[39] T. Kinoshita, T. Wenger, and D. S. Weiss, Observation of a One-Dimensional Tonks-Girardeau Gas, Science 305, 1125 (2004).

[40] B. Paredes, A. Widera, V. Murg, O. Mandel, S. Fölling, I. Cirac, G. V. Shlyapnikov, T. W. Hänsch, and I. Bloch, Tonks-Girardeau Gas of Ultracold Atoms in an Optical Lattice, Nature (London) 429, 277 (2004).

[41] A. H. van Amerongen, J. J. P. van Es, P. Wicke, K. V. Kheruntsyan, and N. J. van Druten, Yang-Yang Thermodynamics on an Atom Chip, Phys. Rev. Lett. 100, 090402 (2008).

[42] H. Spohn and J. L. Lebowitz, Stationary Non-Equilibrium States of Infinite Harmonic Systems, Commun. Math. Phys. 54, 97 (1977).

[43] C. Karrasch, R. Ilan, and J. E. Moore, Nonequilibrium Thermal Transport and Its Relation to Linear Response, Phys. Rev. B 88, 195129 (2013).

[44] B. Doyon, Lower Bounds for Ballistic Current and Noise in Non-Equilibrium Quantum Steady States, Nucl. Phys. B892, 190 (2015).

[45] E. H. Lieb and D. W. Robinson, The Finite Group Velocity of Quantum Spin Systems, Commun. Math. Phys. 28, 251 (1972).

[46] The second inequality in Eq. (3) was shown in Ref. [44], with the goal of establishing the existence of currentcarrying nonequilibrium steady states. The first inequality was suggested by M. J. Bhaseen shortly afterwards (private communication), and can be shown in the same manner.

[47] O. Castro-Alvaredo, Y. Chen, B. Doyon, and M. Hoogeveen, Thermodynamic Bethe Ansatz for NonEquilibrium Steady States: Exact Energy Current and Fluctuations in Integrable QFT, J. Stat. Mech. (2014) P03011.

[48] A. De Luca, J. Viti, L. Mazza, and D. Rossini, Energy Transport in Heisenberg Chains beyond the Luttinger Liquid Paradigm, Phys. Rev. B 90, 161101(R) (2014).

[49] X. Zotos, A TBA Description of Thermal Transport in the XXZ Heisenberg Model, arXiv:1604.08434.

[50] B. Doyon, A. Lucas, K. Schalm, and M. J. Bhaseen, NonEquilibrium Steady States in the Klein-Gordon Theory, J. Phys. A 48, 095002 (2015).

[51] The phrase "local thermodynamic equilibrium", often used to describe the fluid cells, is slightly misleading, as fluid cells generically support nonzero ballistic currents breaking time-reversal symmetry and thus are not at equilibrium. Importantly, in integrable systems, fluid cells are not simply in Galilean or Lorentz boosts of equilibrium states.

[52] C. B. Mendl and H. Spohn, Shocks, Rarefaction Waves, and Current Fluctuations for Anharmonic Chains, arXiv:1607.05205 [J. Stat. Phys. (in press)].
[53] C. Boldrighini, R. L. Dobrushin, and Yu. M. Sukhov, One-Dimensional Hard Rod Caricature of Hydrodynamics, J. Stat. Phys. 31, 577 (1983).

[54] T. Sabetta and G. Misguich, Nonequilibrium Steady States in the Quantum XXZ Spin Chain, Phys. Rev. B 88, 245114 (2013).

[55] T. Antal, P. L. Krapivsky, and A. Rákos, Logarithmic Current Fluctuations in Nonequilibrium Quantum Spin Chains, Phys. Rev. E 78, 061115 (2008).

[56] P. Wendenbaum, M. Collura, and D. Karevski, Hydrodynamic Description of Hard-Core Bosons on a Galileo Ramp, Phys. Rev. A 87, 023624 (2013).

[57] M. Collura and D. Karevski, Quantum Quench from a Thermal Tensor State: Boundary Effects and Generalized Gibbs Ensemble, Phys. Rev. B 89, 214308 (2014).

[58] M. Collura and G. Martelloni, Non-Equilibrium Transport in d-Dimensional Non-Interacting Fermi Gases, J. Stat. Mech. (2014) P08006.

[59] J. Viti, J.-M. Stéphan, J. Dubail, and M. Haque, Inhomogeneous Quenches in a Fermionic Chain: Exact Results, Europhys. Lett. 115, 40011 (2016).

[60] B. Bertini and M. Fagotti, Determination of the Nonequilibrium Steady State Emerging from a Defect, Phys. Rev. Lett. 117, 130402 (2016).

[61] A. Zamolodchikov, Thermodynamic Bethe Ansatz in Relativistic Models. Scaling Three State Potts and LeeYang Models, Nucl. Phys. B342, 695 (1990).

[62] J. Mossel and J.-S. Caux, Generalized TBA and Generalized Gibbs, J. Phys. A 45, 255001 (2012).

[63] L. Bonnes, F. H. L. Essler, and A. M. Läuchli, Light-Cone, Dynamics after Quantum Quenches in Spin Chains, Phys. Rev. Lett. 113, 187203 (2014).

[64] B. Bertini, M. Collura, J. De Nardis, and M. Fagotti, Transport in Out-of-Equilibrium XXZ Chains: Exact Profiles of Charges and Currents, Phys. Rev. Lett. 117, 207201 (2016).

[65] M. Kormos, G. Mussardo, and A. Trombettoni, Expectation Values in the Lieb-Liniger Bose Gas, Phys. Rev. Lett. 103, 210404 (2009).

[66] M. Kormos, G. Mussardo, and A. Trombettoni, OneDimensional Lieb-Liniger Bose Gas as Nonrelativistic Limit of the Sinh-Gordon Model, Phys. Rev. A 81, 043606 (2010).

[67] A. E. Arinshtein, V. A. Fateev, and A. B. Zamolodchikov, Quantum S-Matrix of the $(1+1)$-Dimensional Toda Chain, Phys. Lett. 87B, 389 (1979).

[68] A. V. Mikhailov, M. A. Olshanetsky, and A. M. Perelomov, Two-Dimensional Generalized Toda Lattice, Commun. Math. Phys. 79, 473 (1981).

[69] I. Ya. Aref'eva and V.E. Korepin, Scattering in Two-Dimensional Model with Lagrangian $L=$ $1 / \gamma\left(1 / 2\left(\partial_{m} u u\right)^{2}+m^{2}(\cos u-1)\right)$, Pis'ma Zh. Eksp. Teor. Fiz. 20, 680 (1974).

[70] S. N. Vergeles and V. M. Gryanik, Two-Dimensional Quantum Field Theories which Admit Exact Solutions, Yad. Fiz. 23, 1324 (1976).

[71] B. Schroer, T. T. Truong, and P. H. Weisz, Towards an Explicit Construction of the Sine-Gordon Theory, Phys. Lett. 63B, 422 (1976).

[72] A. B. Zamolodchikov and Al. B. Zamolodchikov, Factorized S-Matrices in Two-Dimensions as the Exact Solutions 
of Certain Relativistic Quantum Field Models, Ann. Phys. (N.Y.) 120, 253 (1979).

[73] M. A. Cazalilla, R. Citro, T. Giamarchi, E. Orignac, and M. Rigol, One Dimensional Bosons: From Condensed Matter Systems to Ultracold Gases, Rev. Mod. Phys. 83, 1405 (2011).

[74] O. A. Castro-Alvaredo, B. Doyon, and T. Yoshimura (to be published).

[75] K. V. Kheruntsyan, D. M. Gangardt, P. D. Drummond, and G. V. Shlyapnikov, Pair Correlations in a Finite-Temperature 1D Bose Gas, Phys. Rev. Lett. 91, 040403 (2003).

[76] C. Destri and H. J. De Vega, New Thermodynamic Bethe Ansatz Equations without Strings, Phys. Rev. Lett. 69, 2313 (1992).

[77] C. Destri and H. De Vega, Unified Approach to Thermodynamic Bethe Ansatz and Finite Size Corrections for Lattice Models and Field Theories, Nucl. Phys. B438, 413 (1995).
[78] I. Bloch, J. Dalibard, and W. Zwerge, Many-Body Physics with Ultracold Gases, Rev. Mod. Phys. 80, 885 (2008).

[79] T. R. Klassen and E. Melzer, The Thermodynamics of Purely Elastic Scattering Theories and Conformal Perturbation Theory, Nucl. Phys. B350, 635 (1991).

[80] A. LeClair and G. Mussardo, Finite Temperature Correlation Functions in Integrable QFT, Nucl. Phys. B552, 624 (1999).

[81] G. Mussardo, Infinite-Time Average of Local Fields in an Integrable Quantum Field Theory after a Quantum Quench, Phys. Rev. Lett. 111, 100401 (2013).

[82] H. Saleur, A Comment on Finite Temperature Correlations in Integrable QFT, Nucl. Phys. B567, 602 (2000).

[83] B. Pozsgay and G. Takacs, Form Factors in Finite Volume II: Disconnected Terms and Finite Temperature Correlators, Nucl. Phys. B788, 209 (2008). 